\title{
Balancing strength and toughness of calcium-silicate-hydrate via random nanovoids and particle inclusions: atomistic modeling and statistical analysis
}

\author{
Ning Zhang ${ }^{1}$ and Rouzbeh Shahsavari ${ }^{1,2,3^{*}}$ \\ ${ }^{1}$ Department of Civil and Environmental Engineering, Rice University, Houston, TX 77005 \\ ${ }^{2}$ Department of Material Science and NanoEngineering, Rice University, Houston, TX 77005 \\ ${ }^{3}$ Smalley Institute for Nanoscale Science and Technology \\ *Corresponding author email: rouzbeh@ rice.edu
}

\begin{abstract}
As the most widely used manufactured material on Earth, concrete poses serious societal and environmental concerns which call for innovative strategies to develop greener concrete with improved strength and toughness, properties that are exclusive in man-made materials. Herein, we focus on calcium silicate hydrate (C-S-H), the major binding phase of all Portland cement concretes, and study how engineering its nanovoids and portlandite particle inclusions can impart a balance of strength, toughness and stiffness. By performing an extensive +600 molecular dynamics simulations coupled with statistical analysis tools, our results provide new evidence of ductile fracture mechanisms in C-S-H - reminiscent of crystalline alloys and ductile metals decoding the interplay between the crack growth, nanovoid/particle inclusions, and stoichiometry, which dictates the crystalline versus amorphous nature of the underlying matrix. We found that introduction of voids and portlandite particles can significantly increase toughness and ductility, specially in $\mathrm{C}-\mathrm{S}-\mathrm{H}$ with more amorphous matrices, mainly owing to competing mechanisms of crack deflection, voids coalescence, internal necking, accommodation, and geometry alteration of individual voids/particles, which together regulate toughness versus strength. Furthermore, utilizing a comprehensive global sensitivity analysis on random
\end{abstract}


configuration-property relations, we show that the mean diameter of voids/particles is the most critical statistical parameter influencing the mechanical properties of $\mathrm{C}-\mathrm{S}-\mathrm{H}$, irrespective of stoichiometry or crystalline or amorphous nature of the matrix. This study provides new fundamental insights, design guidelines, and de novo strategies to turn the brittle C-S-H into a ductile material, impacting modern engineering of strong and tough concrete infrastructures and potentially other complex brittle materials.

Key words: Calcium-silicate-hydrate (C-S-H); Nanovoids; Portlandite particles; Ductile fracture mechanism; Global sensitivity analysis

\section{Introduction}

With more than 30 billion tons per year, concrete manufacturing and use contribute to 5-10\% of $\mathrm{CO}_{2}$ emissions worldwide (Worrell, Price et al. 2001). Among various strategies to reduce this environmental footprint, increasing strength and toughness of concrete from the molecular level up holds a great promise (Qomi, Krakowiak et al. 2014). In this context, understanding and controlling the mechanics of concrete's smallest building block, calcium-silicate-hydrate (C-S$\mathrm{H}$ ), is highly desired but a fundamental and engineering challenge. This is because C-S-H has a variable stoichiometry (Allen, Thomas et al. 2007, Skinner, Chae et al. 2010, Qomi, Krakowiak et al. 2014) and distorted layered structure with multiple defects, porosities, and inclusions (Birchall, Howard et al. 1981, Pellenq, Kushima et al. 2009). Accounting for 60-70\% of cement hydration product, $\mathrm{C}-\mathrm{S}-\mathrm{H}$ is the key structural binder phase in all cement-based materials (Dai, Shui et al. 2012).

For almost all engineering materials, a high damage tolerant, i.e., both strong and tough, is desired but these two properties (strength and toughness) are mutually exclusive except in some biomaterials (Sakhavand and Shahsavari 2015). Strength is the stress that material can resistant 
to non-recoverable deformation, such as plastic deformation in ductile material; whereas, toughness represents the material's resistance to fracture (Ritchie 2011). With growing demand for infrastructure, and also regarding the considerable carbon dioxide emission of cement (contributing to 5-10\% of total anthropogenic $\mathrm{CO}_{2}$ emissions worldwide) (Mehta 2001), the optimization of the mechanical properties and durability of C-S-H has become a pressing need. In the past two decades, great efforts have been undertaken in identifying the role of nanoscale structure (Allen, Thomas et al. 2007, Shahsavari, Buehler et al. 2009, Skinner, Chae et al. 2010, Dolado, Griebel et al. 2011, Shahsavari, Pellenq et al. 2011, Bauchy, Qomi et al. 2014, Qomi, Krakowiak et al. 2014, Ioannidou, Krakowiak et al. 2016), defects (Worrell, Price et al. 2001) and the fracture toughness of C-S-H (Bauchy, Qomi et al. 2014, Qomi, Krakowiak et al. 2014, Bauchy, Laubie et al. 2015). A cement with higher strength leads to more efficient use at smaller quantities (to do more with less) while a higher toughness will extend the ageing of infrastructure, thus reducing maintenance cost and improving global economy. Even a small decrease in cement production imposed by higher strength and/or toughness could have a significant impact in mitigating the global greenhouse $\mathrm{CO}_{2}$ emissions.

When it comes to microstructures with multiple phases or porosity, nucleation, growth, and coalescence of microscopic voids are important ductile fracture mechanisms such as those in metal and alloys (Scheyvaerts, Pardoen et al. 2009, Benzerga and Leblond 2010). In the context of porous cement-based material, $\mathrm{C}-\mathrm{S}-\mathrm{H}$ phase exhibits multiscale porosity ranging from nanometer to millimeter scales (Maekawa 2003). Although the properties of water confined in the gel pores have been well studied experimentally (Greener, Peemoeller et al. 2000, Bordallo, Aldridge et al. 2006, Allen, Thomas et al. 2007, Bauchy, Qomi et al. 2014) and computationally (Jennings 2008, Manzano, Moeini et al. 2012, Gonzalez-Teresa, Dolado et al. 2013), the fracture 
mechanism of porous $\mathrm{C}-\mathrm{S}-\mathrm{H}$ at nanoscale, especially interactions between crack tips and nanovoids, is an important mechanical phenomena, which is essentially unexplored.

Besides C-S-H, about 15-21\% mass of hydrated cement is composed of portlandite, calcium hydroxide $\mathrm{Ca}(\mathrm{OH})_{2}$, making it another important component of concrete (Galmarini, Aimable et al. 2011). The current study of portlandite and its nucleation and growth during hydration of cement mostly focused on micro- and larger scales (Alarcon-Ruiz, Platret et al. 2005, Galmarini, Aimable et al. 2011). Interaction of portlandite and C-S-H at nanoscale, particularly the role of portlandite particles in crack propagation remains unknown. This issue is challenging to tackle by experimental studies alone due to the small scales involved. However, going beyond C-S-H and portlandite, analogous interactions of nanoparticles (Jalilvand and Shahsavari 2015) and shear band in amorphous alloy (Hufnagel, Fan et al. 2002, Kim, Choi et al. 2002, Lewandowski and Greer 2006) has been reported. Mechanisms of absorption, bypassing, cutting, retardation and accommodation were identified experimentally (Glezer, Shurygina et al. 2013). Therefore, an in-depth understanding of portlandite and its interaction with crack tip in C-S-H at nanoscale is very important, and may lead to a better control and tailor the mechanical properties of cement paste and thus concrete properties.

The structure of C-S-H has been comprehensively studied in the last two decades, thanks to the development of research tools including NMR (Cong and Kirkpatrick 1996), XRD (Janik, Kurdowski et al. 2001) and SANS (Allen, Thomas et al. 2007) as well as advanced computational developments (Pellenq, Kushima et al. 2009, Qomi, Krakowiak et al. 2014, Ioannidou, Krakowiak et al. 2016). At the nanoscale, most of the experimental results suggest that $\mathrm{C}-\mathrm{S}-\mathrm{H}$ is analogous to layered crystalline tobermorite and jennite (Cong and Kirkpatrick 1996, Allen, Thomas et al. 2007, Skinner, Chae et al. 2010, Meral, Benmore et al. 2011, 
Gonzalez-Teresa, Dolado et al. 2013). In crystalline C-S-H minerals the interlayer interaction between charged layers is dominated by coulombic interactions rather than van der waals dispersive forces (Shahsavari, Pellenq et al. 2011). A realistic atomistic-level structural model of C-S-H (Pellenq, Kushima et al. 2009) was recently proposed, with which the fundamental deformation mechanism and many other characteristic parameters can be studied. A more complete version of this work was recently developed via a combinatorial approach aimed at computationally generating multiple atomistic $\mathrm{C}-\mathrm{S}-\mathrm{H}$ structures with a wide spectrum of calcium-to-silicon $(\mathrm{C} / \mathrm{S})$ ratios ranging from $\sim 1$ to $\sim 2$ (Qomi, Krakowiak et al. 2014).

In the present work, we aim at understanding the effects of nanovoids and portlandite particles on balancing mechanical properties (strength, stiffness and toughness) of C-S-H under tensile tests using a series of MD simulations coupled with statistical analysis tools. Considering the wide spectrum of $\mathrm{C} / \mathrm{S}$ ratios ( $\sim$ to 2$)$ in cement pastes, we consider three representative $\mathrm{C}-\mathrm{S}-\mathrm{H}$ phases: tobermorite crystal with $\mathrm{C} / \mathrm{S}=1$, and the glassy $\mathrm{C}-\mathrm{S}-\mathrm{H}(\mathrm{gC}-\mathrm{S}-\mathrm{H})$ with $\mathrm{C} / \mathrm{S}=1.565$ and 1.89. Then, random nanovoids and portlandite particles are introduced into the samples and detailed fracture mechanisms and mechanical properties are explored. Besides fundamental understanding, our results will elucidate new fracture mechanisms associated with $\mathrm{C}-\mathrm{S}-\mathrm{H}$ at the molecular scale, particularly the interplay between the crack tip, nanovoid/particle inclusions, and the extent of crystalline/amorphous features of the matrix, thereby providing novel physical insights and guidelines for designing strong and tough cementitious materials.

The paper is organized as follows: section 2 provides a brief introduction of the simulation method, as well as the computer models of tobermorite and $\mathrm{gC}-\mathrm{S}-\mathrm{H}(\mathrm{C} / \mathrm{S}=1.565 ; \mathrm{C} / \mathrm{S}=1.89)$. Implementations of the intra- and inter- layer fractures with random voids and portlandite particles are presented and discussed in section 3. The global sensitivity analysis of the effects of 
microstructure parameters on material properties is presented in section 4, followed by the conclusion of the paper in section 5 .

\section{Methods}

We used molecular dynamics (MD) simulations, which has become an important method to provide an atomistic perspective on structural failure and nanoscale deformation mechanisms of various materials (Zhang, Deng et al. 2011, Zhang and Chen 2013). Successful achievement of any MD modeling requires effective and accurate interatomic potentials. For this purpose, we employed the CSH-FF potential (Shahsavari, Pellenq et al. 2011), which we developed earlier based on extensive ab-initio structural and mechanical data on tobermorite (Shahsavari, Buehler et al. 2009). This force field has been highly successful in predicting accurately structural, elastic, and fracture data of various crystalline and defected C-S-H phases (Shahsavari, Pellenq et al. 2011, Qomi, Krakowiak et al. 2014, Jalilvand and Shahsavari 2015). Throughout this work, constant volume and temperature (NVT) MD simulations are performed. The Nose-Hoover thermostat (Woodcock 1971, Nose 1984) is used to control the temperature of the system, while the velocity - Verlet algorithm (Verlet 1967, Vangunsteren and Berendsen 1990) with a time step of $1 \mathrm{fs}$ is used to integrate the equations of motion. The simulations are performed by using LAMMPS package (Plimpton 1995). 


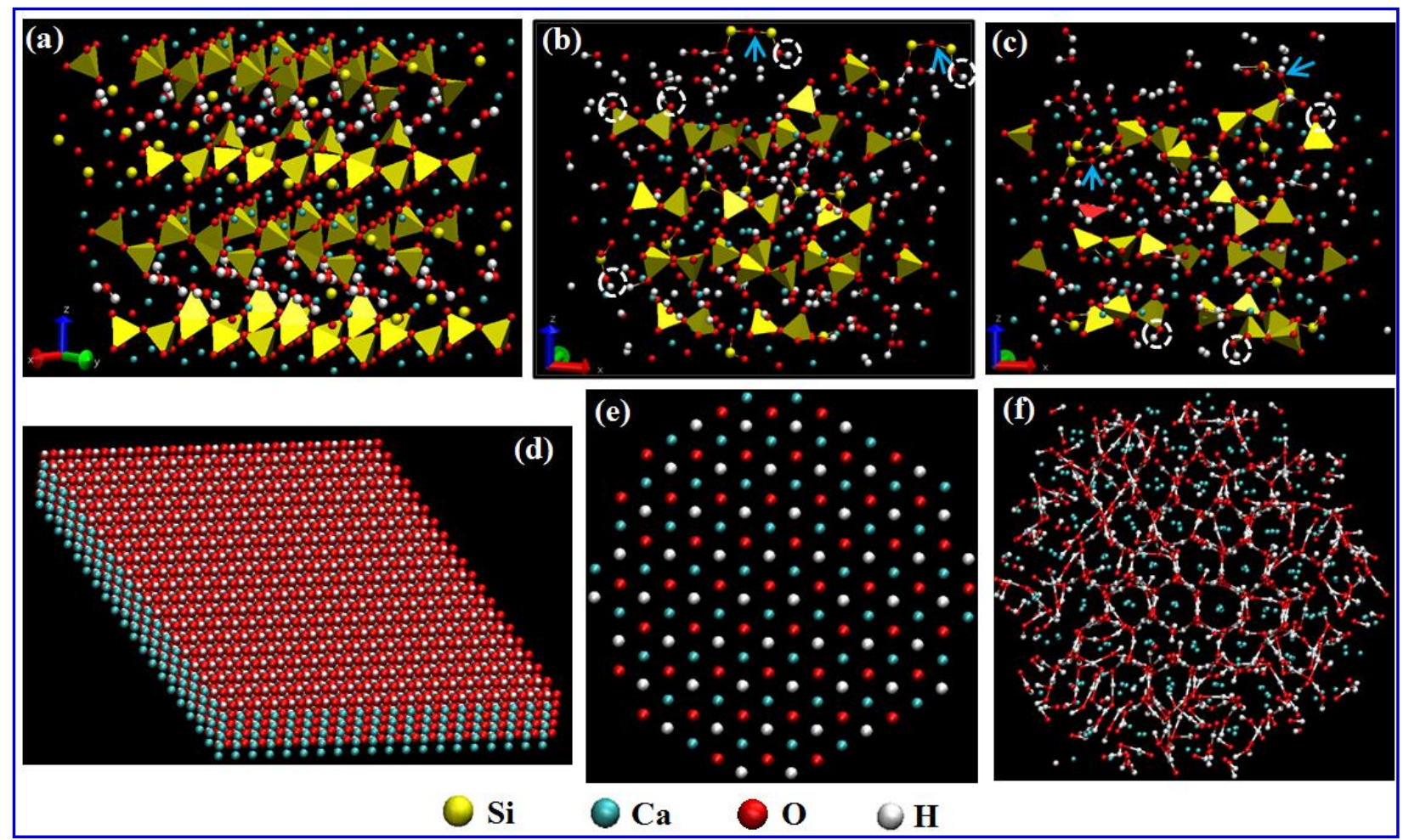

Figure 1. The supercells of (a) tobermorite; (b) $\mathrm{gC}-\mathrm{S}-\mathrm{H}$ with $\mathrm{C} / \mathrm{S}=1.565$; (c) $\mathrm{gC}-\mathrm{S}-\mathrm{H}$ with $\mathrm{C} / \mathrm{S}=1.89$ and $(\mathrm{d})$ portlandite, $\mathrm{Ca}(\mathrm{OH})_{2}$. Top views of atomistic configurations of (e) original and (f) relaxed portlandite particle. The blue arrows in (b-c) indicate the bridging oxygen of two reunited defective silica chains. The white dash circles in (b-c) denote the connection of dissociated protons and terminal oxygen of silica chains.

C-S-H Models. The C/S ratio plays an important role in determining the nanostructure and morphology of C-S-H. A comprehensive procedure to create various combinatorial C-S-H models with a wide range of C/S from 1 to 2 has been reported recently (Birchall, Howard et al. 1981, Qomi, Krakowiak et al. 2014). Here, we will focus on three representative models of C-S$\mathrm{H}$, namely tobermorite as a crystalline analogue of $\mathrm{C}-\mathrm{S}-\mathrm{H}$ with $\mathrm{C} / \mathrm{S}=1.0$ and two defected $\mathrm{C}-\mathrm{S}-\mathrm{H}$ phases with C/S of 1.56 and 1.89 taken from (Qomi, Krakowiak et al. 2014). While the latter two are both semi-crystalline and exhibit a glassy behavior (hereafter called gC-S-H), the C-S-H with 
a higher $\mathrm{C} / \mathrm{S}$ (1.89) has much more defects and $\mathrm{SiO}_{2}$ vacancies, demonstrating an almost amorphous structure (Qomi, Krakowiak et al. 2014) (c.f. Figure 1b-c). In what follows, we describe the atomistic details of these three models.

Crystalline analogue of $\boldsymbol{C}-\mathrm{S}-\mathrm{H}$ : tobermorite. We choose tobermorite $11 \AA$ with $\mathrm{C} / \mathrm{S}=1.0$, whose relaxed lattice parameters are: $a=6.59 \AA, b=7.39 \AA, c=24.38 \AA$ with $\alpha=\beta=90^{\circ}, \gamma=123^{\circ}$, in excellent agreement with the ab-initio and XRD data (Shahsavari, Buehler et al. 2009). A supercell of tobermorite is shown in Figure 1a. It is well known that tobermorite exhibits a clear layered structure, which makes it vulnerable to fail through interlayer fracture. Here, interlayer fracture refers to failure in between the layers while intralayer fracture corresponds to failure within the backbone layers of tobermorite. In this work, crack propagations along both intra- and inter-layer directions of tobermorite are investigated.

To obtain the supercell models of tobermorite, the unit cell is periodically extended along the $x, y$ and $z$ directions. For the cases of intra- and inter-layer fractures, the supercells contain 98,168 and 120,241 atoms with the sizes of $22.0 \times 26.0 \times 2.38 \mathrm{~nm}^{3}$ and $3.0 \times 26.0 \times 22.0 \mathrm{~nm}^{3}$, respectively. In order to induce crack nucleation, an initial notch with length of $5.0 \mathrm{~nm}$ is introduced. Figure 2a presents the original model of tobermorite for interlayer fracture. To study the effect of nanovoids on mechanical properties of tobermorite, random voids with sequential numbers (I, II, III, IV, V) are created (Figure 2b). The voids are generated by removing all atoms within a specified cylindrical region, which is a simplification of the capillary pores in experiment (Feldman and Sereda 1968). After relaxation, although tiny alterations of crystal are observed around the perimeter of the voids, the overall C-S-H structure remains crystalline and well oriented due to the adoption of force field in MD simulations. In this case, the diameters of voids I to $\mathrm{V}$ are selected to be $3.0 \mathrm{~nm}, 1.6 \mathrm{~nm}, 2.4 \mathrm{~nm}, 2.4 \mathrm{~nm}$ and $3.2 \mathrm{~nm}$, respectively. To identify the 
effect of portlandite particles on crack propagation in tobermorite, the random nanovoids are then filled with portlandite particles. For the latter case, the particles are similarly numbered as I, II, III, IV and V (Figure 2c). The nanovoids in our simulations are very similar to the capillary porosity (Chindaprasirt, Jaturapitakkul et al. 2005) rather than gel porosity, which refers to the tiny pores in between the C-S-H lamella (Shahsavari 2011).

Before applying tensile loading, the models are fully relaxed for $0.2 \mathrm{~ns}$ to ensure equilibrium conditions. Thereafter, atomic layers with thickness of $2.0 \mathrm{~nm}$ at both left and right sides of the computer model are held fixed (blue dash frames in Figure 2a), followed by a linear tensile loading on the fixed boundaries. Here, the linear (versus constant tensile loading) is chosen to emulate mode 1 fracture. Otherwise, a constant tensile loading may lead to fracture from initial notch and random voids simultaneously, which is against our objective to investigate the effects of voids and portlandite particles on crack propagation.

Glassy analogues: $g C-S-H(C / S=1.565)$ and $g C-S-H(C / S=1.89)$. During the construction of C$\mathrm{S}-\mathrm{H}$ with various $\mathrm{C} / \mathrm{S}$ ratio, the reactive potential ReaxFF is used for annealing (Qomi, Krakowiak et al. 2014). Since ReaxFF allows for chemical bonds reaction, particularly dissociation of interlayer water molecules to hydroxyl groups and protons, and distortion of the layered silica chains, the final structure of $\mathrm{C}-\mathrm{S}-\mathrm{H}$ is more amorphous than tobermorite. The dissociated protons are found to be connected to the terminal oxygen atoms of silica chain, as denoted by dash circles in Figure 1b-c. Furthermore, the defective silica backbones are observed to reunite through terminal oxygen atoms, as labeled by blue arrows in Figure 1b-c.

Following the procedure of model construction of tobermorite, the supercells of $\mathrm{gC}-\mathrm{S}-\mathrm{H}$ with $\mathrm{C} / \mathrm{S}=1.565$ and 1.89 contain 127,842 and 114,114 atoms, respectively. The size of both gC-S-Hs 
is $3.0 \times 26.0 \times 22.0 \mathrm{~nm}^{3}$. Then same nanovoids and portlandite particles are introduced as those in tobermorite sample for interlayer fracture.

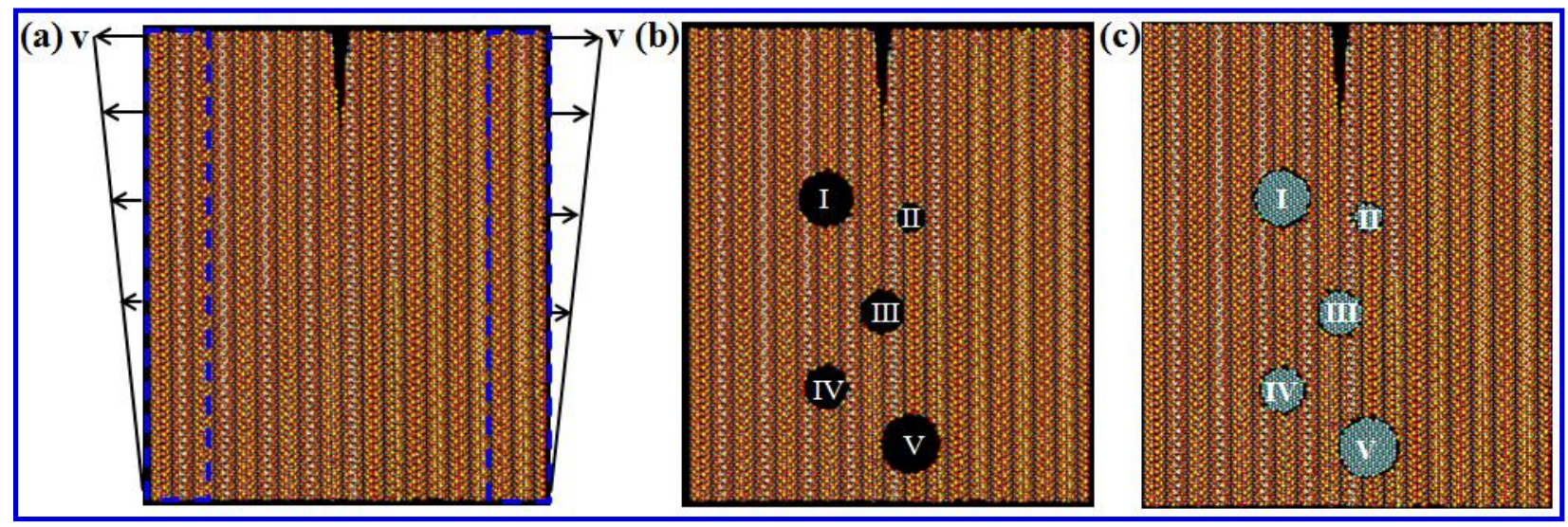

Figure 2. The atomic configurations of tobermorite with (a) original notch; (b) random nanovoids; and (c) portlandite particles. The dash blue frames indicate the fixed boundary regions; the linear uniaxial tensile loading is illustrated by arrows with maximum velocity of $v$ in (a).

\section{Results and Discussions}

In this section, we first use tobermorite to explore intra-layer fracture with a focus on the effects of nanovoids and portlandite particles on mechanical properties, especially toughness (section 3.1). Then interlayer fracture of tobermorite and $\mathrm{gC}-\mathrm{S}-\mathrm{Hs}$ (with $\mathrm{C} / \mathrm{S}=1.565$ and 1.89) are investigated and discussed in sections 3.2, 3.3 and 3.4, respectively. Since the applied tensile loading is linear, the tensile strain is calculated by $(v \times \Delta t \times N) / L$, i.e., the strain at the top of samples. Here $v, \Delta t, N$ and $L$ denote the maximum velocity, timestep, running steps of simulation, and length of the computer model along loading direction, respectively. 


\subsection{Intra-layer fracture of tobermorite}

To begin, we focus on the intra-layer fracture of C-S-H analogues. For the case of pure crack propagation, as shown in Figure 3 (blue line), at the elastic stage, stress increases with strain until reaches its fracture strength of $10.0 \mathrm{GPa}$ at strain $\varepsilon \sim 0.08$. By further increasing the applied strain, a rapid stress drop is observed, which indicates the fracture of material. Consequently, the crack will propagate, and lead to the final rupture of tobermorite, as illustrated by the insets in Figure 3 (points I and J). The stress in Figure 3 is an average stress calculated through the summation of all atoms' forces divided by the perpendicular cross-section area containing the notch (the central plane). 


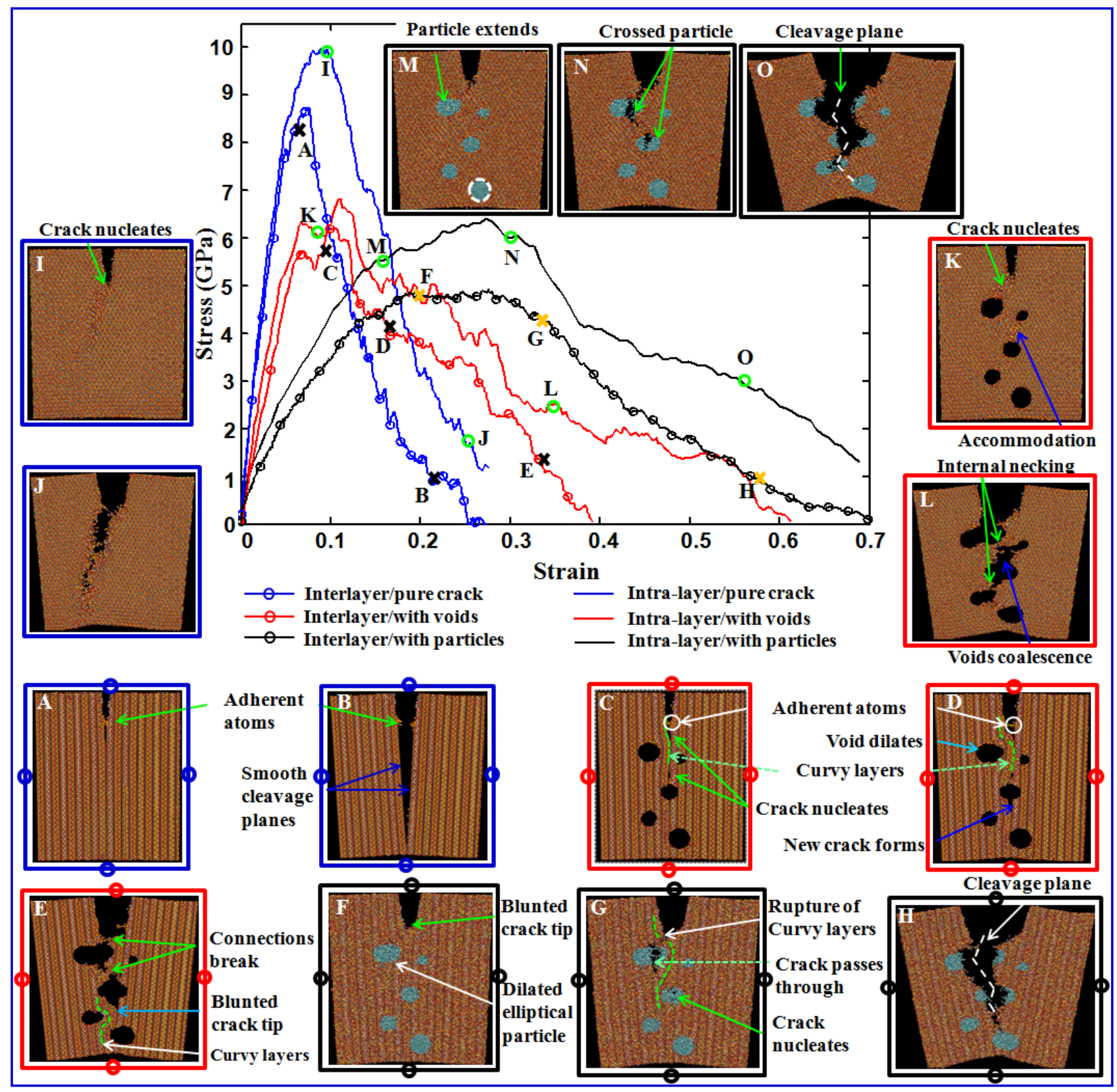

Figure 3. Stress-strain relations for intra-layer and interlayer fracture of tobermorite with pure crack, random nanovoids and portlandite particles under uniaxial tensile loading. The insets illustrate the involved deformation mechanisms during tension. The colored frames and symbols around each atomistic snapshot correspond to the plot legend.

With the introduction of random nanovoids, the path of crack motion will change significantly and the cracks show clear preference to pass through the nanovoids (see snapshots of point $\mathrm{L}$ in 
Figure 3). A slight stress drop is observed at strain $\varepsilon \sim 0.07$ (red line in Figure 3), which corresponds to the initial crack nucleation (see snapshots of point $\mathrm{K}$ in Figure 3). After that, the stress will increase from $6.0 \mathrm{GPa}$ to its fracture strength of $6.8 \mathrm{GPa}$. This is attributed to an important toughening mechanism of accommodation, as identified in nanocrystalline alloys (Glezer, Shurygina et al. 2013). The accommodation mechanism is referred to a phenomenon that a shear band touches a nanoparticles and induces very high elastic stresses in it; then these stresses initiate new shear bands from the edge of nearby particles. In our current work, as the crack nucleates from the tip of the initial notch, an accommodating crack is formed from the edge of void II (snapshots of point $\mathrm{K}$ in Figure 3). Therefore, the crack motion from initial notch across void I, and the one from void II to void III proceed simultaneously. With the increase of tensile strain, the connection between void II and void III breaks, which results in another interesting phenomenon ahead of crack tip, i.e., voids grow to coalescence. Coalescence of microscopic voids is another important failure mechanism in ductile metals and their alloys (Seppala, Belak et al. 2004, Weck, Wilkinson et al. 2008, Benzerga and Leblond 2010, Benzerga and Leblond 2010). Moreover, void coalescence by internal necking that is reported in ductile metals (Scheyvaerts, Pardoen et al. 2009) is also observed in our simulation (snapshot of point L in Figure 3). These phenomena are consistent with the previous studies that $\mathrm{C}-\mathrm{S}-\mathrm{H}$ features a more ductile behavior at nanoscale (Ulm, Bazant et al. 2001, Manzano, Masoero et al. 2013, Bauchy, Laubie et al. 2015). In addition, in this case the geometry of all voids changes from circle to ellipse to absorb more energy during tension. The accommodation mechanism, voids growth and coalescence through internal necking, and the geometry alteration of nanovoids will all synergize to improve toughness. Here, toughness is defined as the amount of energy a material absorbs before failure (representing the work-of-fracture), which is different from the 
classical "fracture toughness" with the unit of $P a \sqrt{m}$. The work-of-fracture is the area under the stress-strain curve, which is deeply affected by gradual, "graceful fracture", whereas the fracture toughness does not incorporate this entire process (Rafiee, Narayanan et al. 2013, Sakhavand, Muthuramalingam et al. 2013, Sakhavand and Shahsavari 2014, Sakhavand and Shahsavari 2015). In addition, stiffness is the slope of the stress-strain curve in the linear elastic region while strength is the maximum stress that the material can withstand.

Next, we fill the nanovoids with portlandite particles. The lattice parameters of portlandite, $\mathrm{Ca}(\mathrm{OH})_{2}$, are: $a=3.5 \AA, b=3.5 \AA, c=4.5646 \AA$ with $\alpha=\beta=90^{\circ}, \gamma=120^{\circ}$. A supercell of portlandite is shown in Figure 1d. We adopt the CSH-FF (Shahsavari, Pellenq et al. 2011) to simulate the atomic interaction of $\mathrm{Ca}(\mathrm{OH})_{2}$. With the introduction of portlandite particles in $\mathrm{C}-\mathrm{S}-\mathrm{H}$ voids, the total energy of simulation system changes significantly. Therefore, a complete relaxation is conducted to achieve the energy minimization. The structure of portlandite particles is observed to change slightly after relaxation (c.f. Figure 1e-f). In view of Figure 1f, the central O-H bonds of $\mathrm{Ca}(\mathrm{OH})_{2}$ particle form hexagonal crystalline structure while the $\mathrm{O}-\mathrm{H}$ bonds around the edge of particle are disordered to form hydrogen bonds with tobermorite. This latter H-bond network act as a connection to glue the two phases (C-S-H and portlandite) together. The less organized shape in Figure 1f compared to the perfect crystalline shape in Figure 1e comes in part from the interfacial interactions of portlandite and C-S-H, which changes the structure locally, and in part from the adopted force field, which lead to minor structural reorientations. Furthermore, the stress-strain curve of the system with portlandite particles becomes more nonlinear with a plateau, which implies more ductile behavior than the systems with pure crack or nanovoids (the yield strain increases from 0.06 in pure tobermorite to 0.27 in tobermorite with portlandte prticles, black line in Figure 3). It can also be seen that akin to the case with voids, crack prefers 
to pass through portlandite particles, and eventually zigzag crack surfaces are observed (see the snapshots of points $\mathrm{M}, \mathrm{N}$ and $\mathrm{O}$ in Figure 3). We note that particle II is bypassed in this fracture simulation, which can be explained by the energy release of crack propagation. Considering the relative positions of void I and void III, the crack between void I and void II is more parallel to the loading direction, which requires more energy for crack propagation. Therefore, crack chooses the path from void I to void III for a faster energy release.

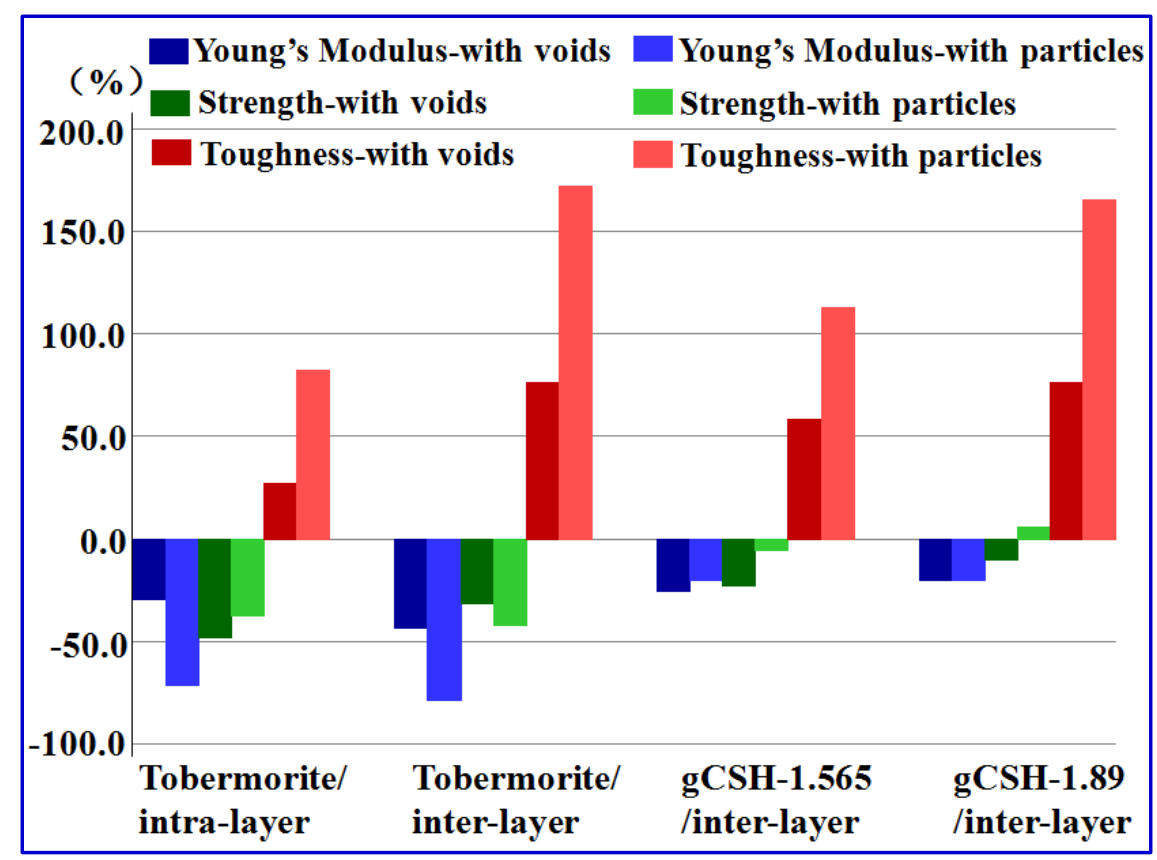

Figure 4. Summary of the effects of nanovoids and portlandite particles on Young's modulus, strength and toughness of tobermorite, gC-S-H $(\mathrm{C} / \mathrm{S}=1.565)$, gC-S-H $(\mathrm{C} / \mathrm{S}=1.89)$ regarding intraand inter-layer fractures. The positive and negative values represent the percentage of increase and decrease of mechanical properties of defective samples (with voids/portlandite particles) with respect to those of defects free samples.

Figure 4 summarizes the effect of voids and portlandite particles on the Young's modulus, strength and toughness of various C-S-H samples. From Figure 3 and Figure 4, we observe that a decrease of Young's modulus goes together with a decrease of strength and an increase of 
toughness of samples with crack, nanovoids and portlandite particles. This is not surprising since Young's modulus seems to be related more to intrinsic values of the individual phases and connectivity (Chamrova 2010). For the case of nanovoids, the decrease of Young's modulus and strength is attributed to the fact that voids act as weak points, i.e. crack nucleation origins. Chemical and physical interactions will occur once the tobermorite with $\mathrm{Ca}(\mathrm{OH})_{2}$ are blended (Figure 1f). Such interactions will have a significant effect on the layered structure of tobermorite, and consequently decrease the Young's modulus and strength, in spite of the relatively large stiffness and strength of both individual phases. However, the toughness of composite will be significantly improved. Indeed, one can approximate from Figure 4 that tobermorite with portlandite particles absorbs $90 \%$ more energy than the one with pure crack propagation, thereby tobermorite with portlandite is a tougher composite.

Although a coupled nanoindentaiton/SEM-EDS study on low water/cement ratio portland cement paste show that portlandite could increase the stiffness of cementitious materials (Chen, Sorelli et al. 2010), the discrepancy between experiment and simulation can come from the following factors :1) in the experiments, the increase of stiffness via inclusion of $\mathrm{CH}$ is attributed to the filling effect of $\mathrm{CH}$ in the gel pores of C-S-H (the tiny pores in between the C-S-H lamella) (Chen, Sorelli et al. 2010). However, this filing effect of gel pores is absent in our simulations and we only considered a bi-phase mixture, 2) the C-S-H in the current work is pure single crystalline material while the C-S-H phase in experiment involves inner and outer C-S-H products as well as defects between grains. Such a structural difference will lead to the discrepancy in the results, and finally 3 ) in the experiment, the length scale is at submicro to micro-meter whereas our simulations is focused at nanometer length scale. 


\subsection{Interlayer fracture of tobermorite}

Considering the layered structure of tobermorite, interlayer fracture is another important concern for engineering researchers. As shown and compared in Figure 3 (blue line with circles), the strength measured from intra-layer fracture is higher than that of interlayer fracture. For the interlayer fracture, the stress-strain curve of pure crack propagation can be divided into three stages: at the elastic stage, stress increases with applied strain linearly until reaches the value of $7.8 \mathrm{GPa}$ at strain $\varepsilon \sim 0.05$. Then the slope of the stress-strain curve drops and the stress approaches the strength of $8.8 \mathrm{GPa}$ at strain $\varepsilon \sim 0.07$. After this yielding point, stress drops rapidly, which indicates a brittle fracture. To identify the underlying deformation mechanism, we track the snapshots of atomic configurations at strains $\varepsilon \sim 0.06$ and $\varepsilon \sim 0.21$ (Figure 3 points $\mathrm{A}$ and B). It is observed that crack nucleates ahead of the adherent atoms. The decrease of slope of the stressstrain curve in the strain range of $0.05 \sim 0.07$ is attributed to the competition of crack nucleation and stretching of adherent atoms. Once crack nucleation is triggered, interlayer brittle fracture will follow, which ultimately leads to the rupture of material. Movie-S1 in supplementary material displays the process of interlayer brittle fracture. The smooth and straight crack surfaces can be observed after failure. The structural character of water molecules, which causes strain localization in the crystal lattice is responsible for such brittle response of tobermorite (Manzano, Masoero et al. 2013).

As for tobermorite with nanovoids, the snapshots of configurations at strains $\varepsilon \sim 0.09,0.16,0.34$ are shown in Figure 3 (points C, D and E). A slight stress drop is observed after the linear elastic range, which corresponds to the initial nucleation of crack. It is also observed that the initially introduced circular nanovoids, such as void I and III, are stretched to become elliptical. From the configuration at strain $\varepsilon \sim 0.09$ (point $\mathrm{C}$ in Figure 3), one can note that nanopores begin to form 
and dilate, which is also responsible for the first stress drop. Then stress increases slightly to its maximum value of $6.1 \mathrm{GPa}$, mainly due to the tension of the adherent atoms ahead of crack tip. After that, the stress drops gradually. An interesting phenomenon is that the original straight layer has been stretched to become curvy. Compared to the brittle fracture in the case of tobermorite with pure crack, the crack tip is blunted and deflected due to the perturbation of nanovoids. Another interesting finding is that a new crack is nucleated from the edge of void III (see snapshot of point D in Figure 3). However, with continued loading, the tip of this newly formed crack is also blunted and the propagation is impeded due to the participation of voids IV and V. Simultaneously, the initial crack goes through voids I and III (see snapshot of point E in Figure 3). The detailed processes of such crack deflection is presented in the supplementary Movie-S2. Considering the relative locations of void I to void II and void I to void III, crack chooses the path from void I to void III for a faster energy release. Therefore, void II is bypassed again as discussed earlier for the case of intra-layer fracture.

With the addition of portlandite particles, the stress-strain curve shows a totally different pattern with respect to the samples with pure crack and nanovoids (black line with circles in Figure 3). At the initial stage, stress increases with strain nonlinearly until it reaches a plateau at a relatively large strain $\varepsilon \sim 0.2$. As the tensile strain increases to $\varepsilon \sim 0.3$, stress drops very slowly until the failure of the composite. To identify the deformation mechanisms, we carefully analyzed the snapshots of the atomic configurations at strains $\varepsilon \sim 0.2,0.35,0.57$ in Figure 3 (points F, G and H) (Movie-S3). The crack tip has become very blunt before cutting through particle I, and the original circular particle I is stretched to become elliptical. The curvy layered structure is stretched to break during tension. To choose a path for faster energy release, crack crosses particles I, III and V, and bypasses particles II and IV, leaving a zigzag crack surfaces. In view of 
the stress-strain curve in Figure 3, the addition of portlandite particles has greatly increased the toughness of tobermorite, in spite of the decrease in strength. The mode of failure has transferred from brittle to ductile due to the large fracture strain and rather long and smooth stress plateau.

Similar to the intra-layer fracture, a decrease of Young's modulus goes with a decrease of strength but an increase of toughness, as summarized in Figure 4. This can be explained by the intrinsic properties of the materials. The introduction of voids is equivalent to adding weak spots into material, which then leads to the decrease of stiffness and strength. However, crack propagation is deflected by the voids, and consequently more energy is absorbed during fracture, thereby improving toughness. The dominated interlayer interaction in crystalline tobermorite is coulombic interactions (Shahsavari, Pellenq et al. 2011). Once the voids are filled with portlandite particles, structural heterogeneity appears, which entail new interfacial chemical interactions involving hydrogen bonds and van der waals interactions, hence, reducing the dominant role of coulombic interactions. It is well known that coulombic interaction is much stronger than hydrogen bonds or van der waals interactions (Zhang and Chen 2012). Therefore, while structural heterogeneity and crack deflection imposed by portlandite particles decrease the Young's modulus and strength they increase the toughness due to tortuosity of crack path.

\subsection{Interlayer fracture of $\mathrm{gC}-\mathrm{S}-\mathrm{H}(\mathrm{C} / \mathrm{S}=1.565)$}

To systematically evaluate the role of nanovoids and portlandite particles in balancing the strength and toughness of C-S-H with various compositions, in this section, we turn our attention to $\mathrm{gC}-\mathrm{S}-\mathrm{H}$ with $\mathrm{C} / \mathrm{S}=1.565$. As seen in Figure 5, the stress-strain curve of $\mathrm{gC}-\mathrm{S}-\mathrm{H}$ becomes more nonlinear than that of tobermorite (see inset in Figure 5a). For the $\mathrm{gC}-\mathrm{S}-\mathrm{H}$ with pure crack, the interlayer fracture strength is $4.0 \mathrm{GPa}$ at strain $\varepsilon \sim 0.04$, which is $55 \%$ lower than that of tobermorite. This observation is consistent with the previous reports of yield strength for 
tobermorite and gC-S-H (Manzano, Masoero et al. 2013, Qomi, Krakowiak et al. 2014). By further increasing the strain, the stress gradually drops until the rupture of material. However, the Young's modulus of $\mathrm{gC}-\mathrm{S}-\mathrm{H}(\mathrm{C} / \mathrm{S}=1.565)$ is about $20 \%$ higher than that of tobermorite. This can be intuitively expected because the elastic moduli is expected to increase with the average density of atoms (Manzano, Masoero et al. 2013), which is higher for gC-S-H, i.e., 65 atoms/nm ${ }^{3}$ for tobermorite vs. 91 atoms $/ \mathrm{nm}^{3}$ for $\mathrm{gC}-\mathrm{S}-\mathrm{H}(\mathrm{C} / \mathrm{S}=1.565)$. To characterize the deformation mechanisms, atomistic configuration at strains $\varepsilon \sim 0.16$ is shown in Figure 5 (point A). It should be noted that the relaxed structure of $\mathrm{gC}-\mathrm{S}-\mathrm{H}(\mathrm{C} / \mathrm{S}=1.565)$ is more amorphous with respect to the crystalline tobermorite. As stated in section 3.2, this amorphous structure is due to the strategy of model construction via breaking continuous silica chains. Moreover, interlayer fracture of gC-S$\mathrm{H}(\mathrm{C} / \mathrm{S}=1.565)$ seems to be more ductile than tobermorite. This is attributed to the distorted layers of gC-S-H, which cause interfacial atoms adhere on the crack surfaces. Therefore, as the material eventually ruptures, rough crack surfaces are left. 


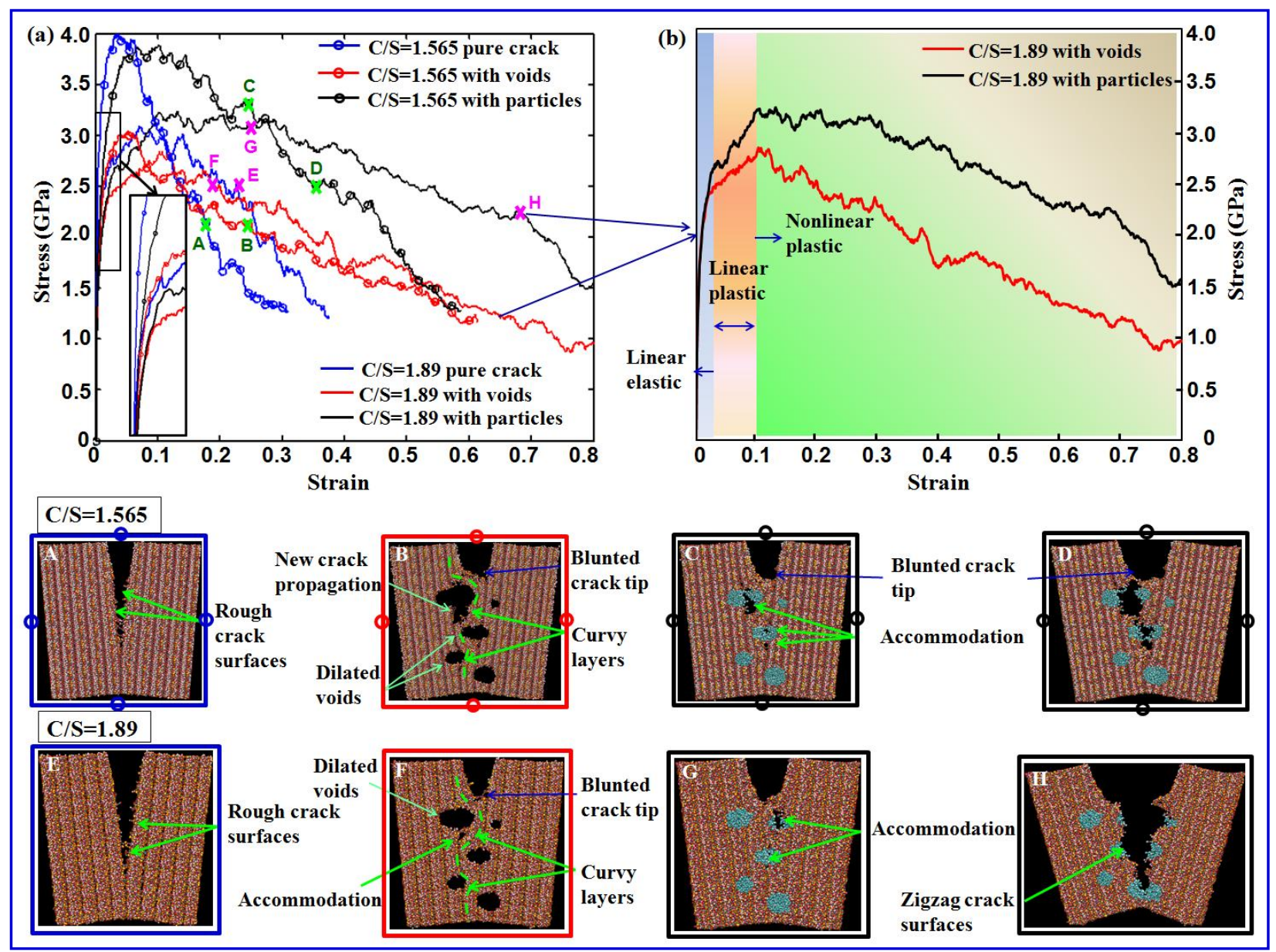

Figure 5. (a) Comparison of stress-strain relations for interlayer fracture in $\mathrm{gC}-\mathrm{S}-\mathrm{H}(\mathrm{C} / \mathrm{S}=1.565)$ and $\mathrm{gC}-\mathrm{S}-\mathrm{H}(\mathrm{C} / \mathrm{S}=1.89)$ in terms of with pure crack, nanovoids and portlandite particles under uniaxial tensile loading. The inset shows the initial part of stress-strain curve. (b) stress-strain curves of $\mathrm{gC}-\mathrm{S}-\mathrm{H}(\mathrm{C} / \mathrm{S}=1.89)$ with voids and portlandite particles. The blue, orange and green shadows represent the three different stages of deformation. The colored frames and symbols around each atomistic snapshot correspond to the plot legend.

$\mathrm{gC}-\mathrm{S}-\mathrm{H}(\mathrm{C} / \mathrm{S}=1.565)$ specimen with nanovoids follows a similar behavior to the case without voids, however, it can be noted from Figure 5a and Figure 4 that although the strength is weakened by $17.5 \%$, nanovoids absorb $36.7 \%$ more energy during fracture and thus improve the toughness of $\mathrm{gC}-\mathrm{S}-\mathrm{H}$. The snapshot of $\mathrm{gC}-\mathrm{S}-\mathrm{H}$ with nanovoids at strains $\varepsilon \sim 0.25$ is presented in 
Figure 5 (point B). Compared to the gC-S-H without voids, the crack tip is observed to be blunted prior to propagation and the initial straight layer is bent. With continued loading, the shape of voids I, III and IV changes from circle to ellipse, through which more energy is absorbed and crack propagation is postponed. This is a key process that contributes to the improved toughness. In addition, a new crack forms from the edge of void I and eventually breaks the connection between voids I and III. The shape of voids II and V is not changed, and thus these two voids are bypassed. The path of fast energy release rate in this case is along voids I, III and IV.

With the addition of portlandite particles, the fracture toughness is further improved by nearly $2 \mathrm{X}$ with a slight sacrifice of strength and Young's modulus with respect to the case with pure crack propagation. Tracking the process of deformation at strains $\varepsilon \sim 0.23$ and 0.35 (Figure 5 points $\mathrm{C}$ and $\mathrm{D}$ ), it is observed that the crack tip is blunted dramatically before crossing void I. Nanopores are observed to form nearby portlandite particles. Failure mechanism of accommodation is also observed. New cracks are imparted from void III before the initial crack passes through void I. It is worthy to mention that fracture takes place through cutting the portlandite particles rather than splitting the grain boundaries, which indicates a strong interaction between these two material phases. Eventually, crack is deflected along the fast energy release rate path of particles I, III and V. In contrast, no deformation of particles II and IV is observed and these two particles are bypassed.

\subsection{Interlayer fracture of $g C-S-H(C / S=1.89)$}

Focusing on another chemistry of C-S-H, it can be noted from the stress-strain curves in Figure 5 that the toughness of $\mathrm{gC}-\mathrm{S}-\mathrm{H}$ with $\mathrm{C} / \mathrm{S}=1.89$ has been significantly improved due to the participation of nanovoids and portlandite particles. Moreover, akin to $\mathrm{gC}-\mathrm{S}-\mathrm{H}(\mathrm{C} / \mathrm{S}=1.565)$, the 
failure mode of $\mathrm{gC}-\mathrm{S}-\mathrm{H}(\mathrm{C} / \mathrm{S}=1.89)$ with pure crack is also much more ductile than that of tobermorite due to the amorphous structure of $\mathrm{gC}-\mathrm{S}-\mathrm{H}$. The overall pattern of stress-strain curves is nonlinear. The Young's modulus of $\mathrm{gC}-\mathrm{S}-\mathrm{H}(\mathrm{C} / \mathrm{S}=1.89)$ is smaller than that of $\mathrm{gC}-\mathrm{S}-\mathrm{H}$ $(\mathrm{C} / \mathrm{S}=1.565)$, due to the decrease of local atom density, i.e. 91 atoms $/ \mathrm{nm}^{3}$ for $\mathrm{gC}-\mathrm{S}-\mathrm{H}$ $(\mathrm{C} / \mathrm{S}=1.565)$ vs. 81 atoms $/ \mathrm{nm}^{3}$ for $\mathrm{gC}-\mathrm{S}-\mathrm{H}(\mathrm{C} / \mathrm{S}=1.89)$. For pure crack propagation, the strength is measured to be $3.1 \mathrm{GPa}$ at strain $\varepsilon \sim 0.06$. The snapshot at strain $\varepsilon \sim 0.25$ (Figure 5 point E) reveals that the adherence of atoms in between the layers inhibits the crack propagation, which consequently leads to the nonlinearity and fluctuation of stress-strain curve (Figure 5). Such characteristics of stress-strain curve were also observed in shear simulations of gC-S-H (Manzano, Masoero et al. 2013). Furthermore, rough cleavage surfaces are left after fracture (Figure 5 point E). The chemical bonds interactions between layers including hydrogen bonds and van der waals interactions are believed to play a key role in brittle to ductile transition of $\mathrm{gC}$ S-H.

With the introduction of nanovoids, $\mathrm{gC}-\mathrm{S}-\mathrm{H}(\mathrm{C} / \mathrm{S}=1.89)$ becomes more ductile but with a slight decrease in fracture strength. In this case, we divide the stress-strain curve into three stages. Specifically, we plot the stress-strain relation of $\mathrm{gC}-\mathrm{S}-\mathrm{H}(\mathrm{C} / \mathrm{S}=1.89)$ with nanovoids and portlandite particles in Figure 5b. At low strain (up to 0.025), the mechanical response of gC-S-H $(\mathrm{C} / \mathrm{S}=1.89)$ sample with nanovoids is linear elastic with stress till $\sim 2.5 \mathrm{GPa}$ (blue shadow in Figure 5b). Afterwards, the slope of stress-strain curve decreases and stress increases gradually to $2.8 \mathrm{GPa}$ at strain of 0.12 (orange shadow in Figure $5 \mathrm{~b}$ ). This is attributed to the competition between crack nucleation and resistance of adherent atoms ahead of crack tip. It is noted from the atomistic configuration in Figure 5 (point F) that crack tip is blunted and the original straight layer has been stretched to bend. As the critical strain of 0.12 , the adherent atoms are completely 
separated. Then crack is deflected to void I, and meanwhile a new nanopore is found to form beneath void I. The geometry of voids I, III, IV changes from original circular shape to ellipse. All of these deformation mechanisms will make contributions to increase toughness. With continued loading, voids I and III are connected ultimately through internal necking, which is a common fracture mechanism observed in ductile metals (Scheyvaerts, Pardoen et al. 2009).

Considering $\mathrm{gC}-\mathrm{S}-\mathrm{H}(\mathrm{C} / \mathrm{S}=1.89)$ with portlandite particles, surprisingly, the toughness is significantly improved without any sacrifice of strength. Particularly, compared to the case of pure crack propagation, a slight increase of strength is observed in the stress-strain curve. At the strain smaller than 0.03, the mechanical response is linear elastic (blue shadow in Figure 5b). After that one can note a decrease of gradient of stress-strain curve and the fluctuation of stress, which is akin to the case with nanovoids (orange shadow in Figure 5b). Competition of crack tip motion and stretching of adherent atoms in front of the crack tip is responsive for this decrease of slope of the stress-strain curve. Then, an oscillated stress plateau is observed from the strain of 0.1 to 0.28 (green shadow in Figure $5 \mathrm{~b}$ ). This is attributed to the competition of original crack tip blunting and new crack nucleation from particle II and III (see snapshots of point $\mathrm{G}$ and $\mathrm{H}$ in Figure 5). Subsequently, the original crack motion is arrested due to the extension of portlandite particles and formation of nanopores. However, with the increase of the external load, the connection between original crack and particle II is broken, which corresponds to the stress drop at strain $\varepsilon \sim 0.28$. Ultimately, the system ruptures by cutting through portlandite particles. Crack deflection, combined with the deformation of portlandite particles, absorb remarkable energy during fracture, and thus make the toughness of composite significantly higher than each of its components: gC-S-H and portlandite. It is worth mentioning that particle I, which is larger than particle II and usually has the priority to deflect crack motion than particle II, is bypassed in this 
simulation case. This implies that crack deflection path has a complex dependency on the size of particle and the chemistry of the underlying matrix (the extent of amorphous vs. crystalline nature of C-S-H). Therefore, it is necessary to statistically analyze the relation between these inhomogeneous structural patterns and mechanical properties.

\section{Statistical sensitivity analysis}

Ductile fracture of C-S-H depends strongly on the size and location of voids and portlandite particle inclusions. In this section, statistical sensitivity analysis techniques are utilized to study the effect of random nanostructural configurations on mechanical properties. Such information is crucial to quantify the reliability of aforementioned computational analysis in predicting the material performance and potentially developing design guidelines. To align our focus in this section, we consider the interlayer fracture of tobermorite and $\mathrm{gC}-\mathrm{S}-\mathrm{H}(\mathrm{C} / \mathrm{S}=1.56$ and 1.89) with addition of nanovoids and portlandite particles. In order to compare the relative effect of inclusions (versus nanovoids) on mechanical properties, we considered all structures with random nanovoids and only filled their voids with the inclusions. In this way, any property changes are solely due to the filling effect of inclusions and not the changes in geometry.

The variance-based global sensitivity analysis (GSA) method, which investigates the contributions of input variables (e.g., particle diameter) to the variance of an output variable (e.g. strength), is adopted in this study. According to the concept of variance-based GSA, the total variance $\mathrm{V}$ of an output function $\mathrm{f}(\mathrm{X})$, such as strength, is decomposed into various sources of input variable variations (Yin, Chen et al. 2008),

$$
V=\sum_{i} V_{i}+\sum_{i<j} V_{i j}+\cdots+V_{12 \ldots m}
$$


where $m$ is the number of input variables; $V_{i}$ represents the partial variance in the response due to the main effect of an input variable $X_{i}$ alone. The higher order terms represent the interaction effects between two or more input variables. In this study, both the main effect and the total effect, which is referred as the sum of main and interaction effects, are investigated. The global sensitivity index reads $M S I_{i}=V_{i} / V, S I_{i j}=V_{i j} / V$, and so on. The total sensitivity index is the measure of importance of an input variable, and is defined as the sum of all sensitivity indices that involve the input variable of interest, including both the main effect and the effects due to interactions with other input variables. Thus, by applying GSA, we expect to uncover the significance of each input variable as well as their complex interactions with other input types on output responses (e.g. strength, toughness and so on). This will likely enable de novo strategies to understand and tune the configuration of C-S-H matrix with voids/particle inclusions.

\subsection{Global sensitivity analysis of tobermorite with voids}

To examine the relation between random nanostructure parameters and mechanical properties, a random configuration generator is developed to provide multiple nanostructure configurations based on a set of basic parameters. In this context, it is important to realize and determine the critical nanostructure descriptors that are relevant to our system in a statistical sense. For the present study, a basic set of nanostructure parameters is illustrated in Figure 6. In view of the ductile fracture induced by the interaction between initial crack and voids, the diameter $(d)$ and location of voids are set as two basic parameters. The critical location of voids is determined by its nearest neighbor void distance, $N N D$. Moreover, considering the interlayer brittle fracture (Figure 3), the distance from the center of voids to the central line of model, $d_{\text {central }}$, will be another important structural descriptor. In statistics, standard deviation (STD) is a crucial measure that is used to quantify the amount of variation or dispersion of a set of data. Hence, 
STDs of nanostructure parameters, $d, N N D, d_{\text {central }}$, are also included as structural descriptors to study the relation of random configuration-mechanical property in a large phase space of parameters. Table 1 lists these key six nanostructure parameters considered in this paper. As the inputs to the random configuration generator, we set the number of voids $(N)$ to 5 , and the minimum allowed distance between edges of voids $(N N D)$ to $3.0 \AA$ to avoid coalescence in the initial configurations. Moreover, the distance between nanovoids and the boundary of model is set to be at least $3.0 \mathrm{~nm}$, while the distance between the nanovoids and the original notch tip is set to be at least $2.0 \mathrm{~nm}$. The diameter $(d)$ and location of voids $\left(d_{\text {central }}\right)$ are randomly assigned during model construction and the maximum diameter is set to be $40.0 \AA$.

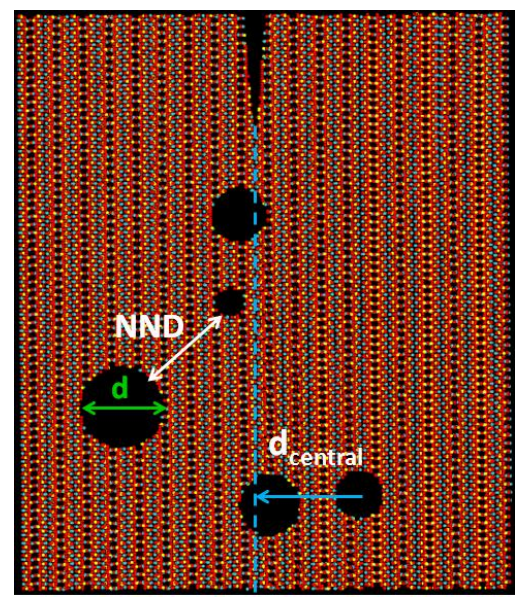

Figure 6. Illustration of nanostructure parameters for statistical analysis of multiple configurations. $d, N N D$, and $d_{\text {central }}$ represent the diameter of void, minimum distance between two voids and the distance between center of void and central line of crack notch.

Table 1. Nanostructure parameters for statistical analysis of multiple configurations.

\begin{tabular}{ll}
\hline Symbol & Description \\
\hline cent.mean & Mean of central distance \\
cent.std & Standard deviation of central distance \\
\hline
\end{tabular}




\begin{tabular}{ll}
\hline diam.mean & Mean of diameter \\
diam.std & Standard deviation of diameter \\
NND.mean & Mean of nearest neighbor distance \\
NND.std & Standard deviation of nearest neighbor distance \\
\hline
\end{tabular}

As the first step to have meaningful statistical results, the sufficient number of random configurations should be determined. Figure 7 presents the convergence of the mean and standard deviation of Young's modulus, strength and toughness of tobermorite containing random voids/ particles as a function of number of simulations. As an example, the mean of Young's modulus, strength and toughness of tobermorite with portlandite particles will converge within a $\pm 1 \%$ error bounds after 49,56 and 85 simulations while their standard deviations converge after 57, 56 and 55 simulations, respectively. As a conservative choice, we select 100 simulations to represent sufficient random configurations in this work. Figure 8a presents three representative random nanostructure configurations and their corresponding mechanical responses. The stress-strain curves obtained from a set of $100 \mathrm{MD}$ simulations of tobermorite with voids are shown in Figure 8b. We note that although all these configurations have the same number of voids $(N=5)$, minimum allowed distance $(3.0 \AA)$ and maximum allowed diameter (40.0 ̊), the models exhibit various stress-strain relations. This observation implies that the random structural features have important effects on mechanical properties. We consider Young's modulus, strength and toughness as the response variables of interests. The variance of the main effects and interaction effects (up to the second order) of parameters for tobermorite are listed in Table 2. For example, ISI of cent.mean (V1) includes all two-body interactions that have $V 1$, i.e. $V 1 V 2+V 1 V 3+V 1 V 4+V 1 V 5+V 1 V 6$. The larger MSI (or ISI), the more importance the effect of that parameter(s) on the output response. The main sensitivity index (MSI), i.e. $V_{i} / V$, 
and interaction sensitivity index (ISI) of the critical nanostructure parameters of tobermorite with voids or portlandite particles are shown in Figure 9a,b, which clearly indicates that the relative impacts of the nanostructure parameters on the mechanical properties are different.

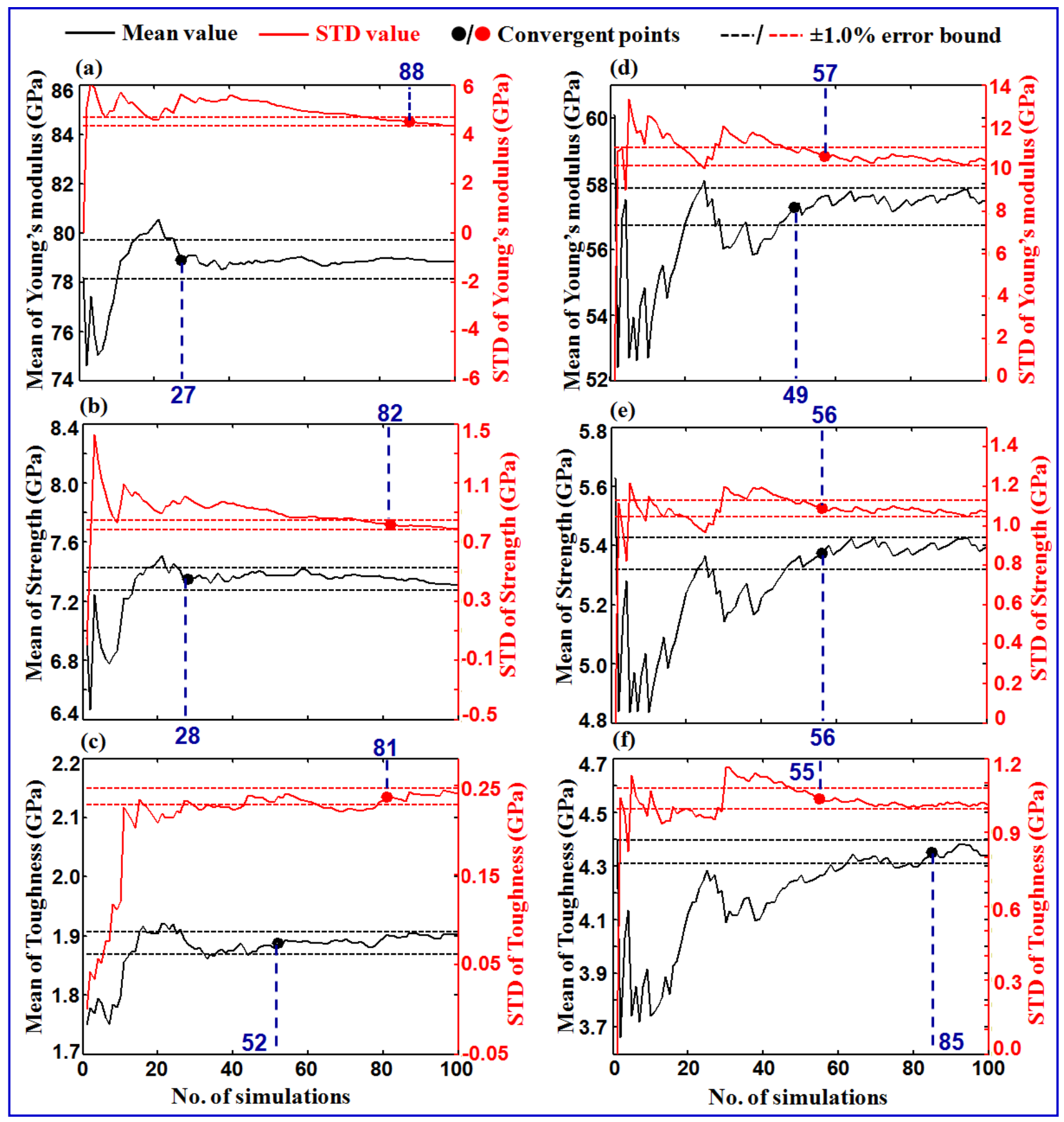


Figure 7. Illustration of convergence of mean and standard deviation of Young's modulus, strength and toughness in terms of the number of simulations. (a-c) and (d-f) correspond to the cases of tobermorite samples with random voids and portlandite particles, respectively.

Table 2. Summary of Main and interaction effects of multiple microstructure parameters on Young's modulus, strength and toughness of tobermorite with random voids and random portlandite particles.

\begin{tabular}{|c|c|c|c|c|c|c|c|}
\hline \multirow[t]{2}{*}{ Index } & \multirow[t]{2}{*}{ Input } & \multicolumn{2}{|c|}{$\begin{array}{c}\text { Variance of Young's } \\
\text { modulus }(\%)\end{array}$} & \multicolumn{2}{|c|}{$\begin{array}{c}\text { Variance of strength } \\
(\%)\end{array}$} & \multicolumn{2}{|c|}{$\begin{array}{c}\text { Variance of toughness } \\
(\%)\end{array}$} \\
\hline & & Voids & Particles & Voids & Particles & Voids & Particles \\
\hline \multirow{5}{*}{$\begin{array}{l}\text { Main } \\
\text { effect }\end{array}$} & $\mathrm{V} 1$ & 2.98 & 3.51 & 3.72 & 3.30 & 13.95 & 7.73 \\
\hline & $\mathrm{V} 2$ & 1.24 & 3.50 & & & 2.66 & 2.19 \\
\hline & V4 & $\frac{40.85}{3.99}$ & 0.75 & 4.55 & 0.80 & 2.60 & 0.63 \\
\hline & V5 & 2.87 & 3.05 & 4.08 & 2.81 & 2.23 & 2.67 \\
\hline & V6 & 2.20 & 2.92 & 6.85 & 3.74 & 3.35 & 3.23 \\
\hline \multirow{15}{*}{$\begin{array}{l}\text { Interaction } \\
\text { effect }\end{array}$} & $\mathrm{V} 1 . \mathrm{V} 2$ & 1.09 & 5.95 & 1.65 & 3.19 & 1.70 & 2.93 \\
\hline & V1.V3 & 0.86 & 3.48 & 0.95 & 4.64 & 0.14 & 2.38 \\
\hline & V1.V4 & 0.61 & 0.00 & 0.67 & 0.00 & 0.20 & 0.00 \\
\hline & V1.V5 & 3.95 & 1.51 & 4.73 & 1.65 & 0.38 & 0.99 \\
\hline & V1.V6 & 1.42 & 0.27 & 1.79 & 0.32 & 1.62 & 0.48 \\
\hline & $\mathrm{V} 2 . \mathrm{V} 3$ & 0.15 & 2.94 & 0.17 & 2.79 & 0.47 & 2.65 \\
\hline & $\mathrm{V} 2 . \mathrm{V} 4$ & 0.08 & 0.00 & 0.11 & 0.00 & 0.66 & 0.00 \\
\hline & V2.V5 & 0.67 & 1.34 & 1.15 & 1.01 & 1.17 & 1.17 \\
\hline & V2.V6 & 0.22 & 0.24 & 0.37 & 0.21 & 5.51 & 0.62 \\
\hline & V3.V4 & 0.06 & 0.00 & 0.06 & 0.00 & 0.05 & 0.00 \\
\hline & V3.V5 & 0.46 & 0.82 & 0.46 & 1.57 & 0.09 & 0.95 \\
\hline & V3.V6 & 0.16 & 0.14 & 0.19 & 0.30 & 0.44 & 0.47 \\
\hline & V4.V5 & 0.31 & 0.00 & 0.34 & 0.00 & 0.13 & 0.00 \\
\hline & V4.V6 & 0.10 & 0.00 & 0.13 & 0.00 & 0.60 & 0.00 \\
\hline & $\mathrm{V} 5 . \mathrm{V} 6$ & 0.74 & 0.06 & 0.96 & 0.11 & 1.10 & 0.22 \\
\hline
\end{tabular}

Note: V1, V2, V3, V4, V5, V6 in Table 2, 3, 4 represent the parameters of cent.mean, cent.std, diam.mean, diam.std, NND.mean and NND.std, respectively. 

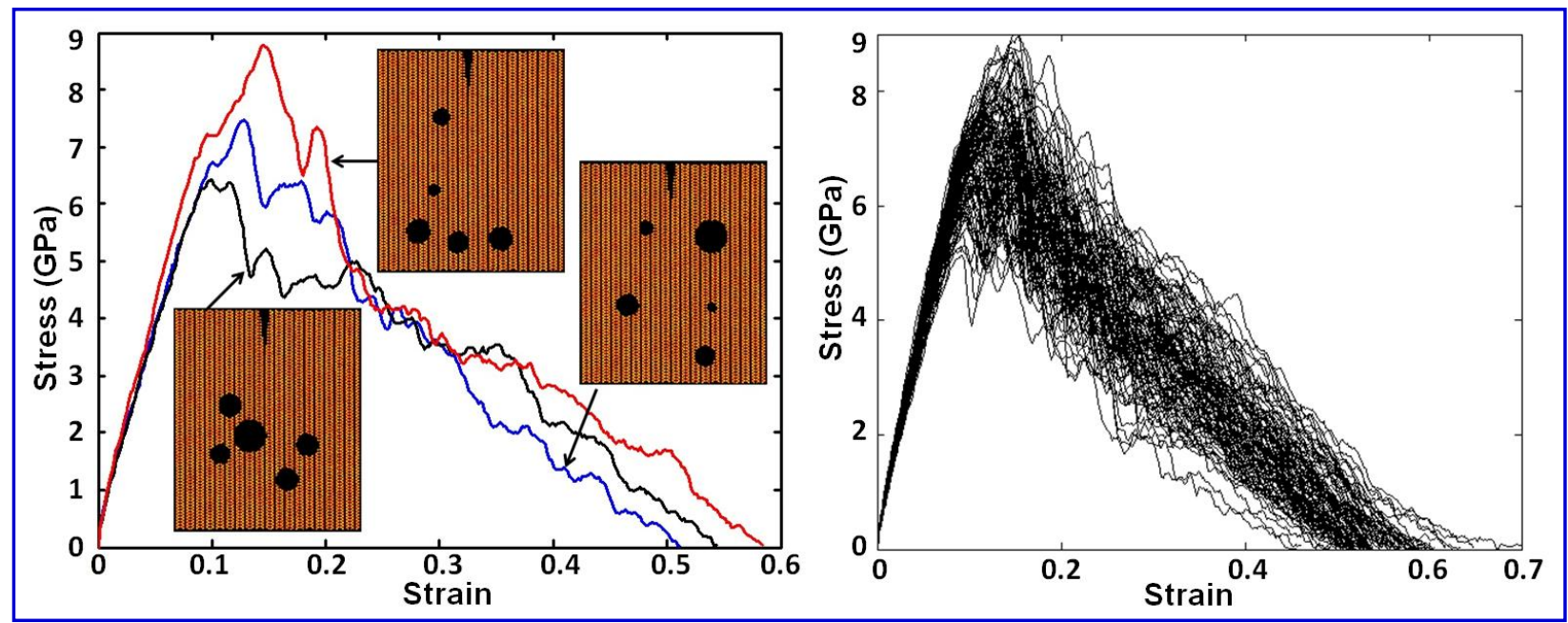

Figure 8. (a) Three random configurations and their corresponding stress-strain curves. (b) A combination of stress-strain curves obtained from 100 simulations.

Among the six input parameters, the main effect of diam.mean (the mean diameter of void), V3 in Table 2, is found to be the most statistically significant input parameter for the three output mechanical responses, particularly for Young's modulus (Figure 9a). For toughness, the main effect of cent.mean (the mean of central distance), VI in Table 2, makes equivalent vital contribution as the main effect of diam.mean. Generally, the contributions of the main effects of input parameters are much larger than their interaction effects, particularly for $V 3$ and $V 1$. Furthermore, for Young's modulus and strength, the interaction effect of cent.mean and NND.mean (V5), the mean of nearest neighbor distance, (V1.V5 in Table 2) is larger than their individual main effects (VI or V5). Another interesting observation from Figure 9a is that the interaction of cent.std (V2) and NND.std (V6), (V2.V6 in Table 2), which relates to the scattering of neighboring voids nearby the central line, has a remarkable impact on toughness. The more this scattering, the larger the toughness. This is because cent.std is a critical precursor for crack propagation through voids, while NND.std relates to the probability of voids coalescence. Tuning 
these two parameters will significantly impact the crack propagation path and thus the required energy for crack growth (i.e. toughness). In contrast, the diam.std (V4) makes relatively small contribution to mechanical properties, either in the form of MSI or ISI; i.e. for a given average void size, its scatter is not so important. These findings are somewhat non-intuitive and only possible via such accurate statistical analysis performed on our hundreds of MD simulations.

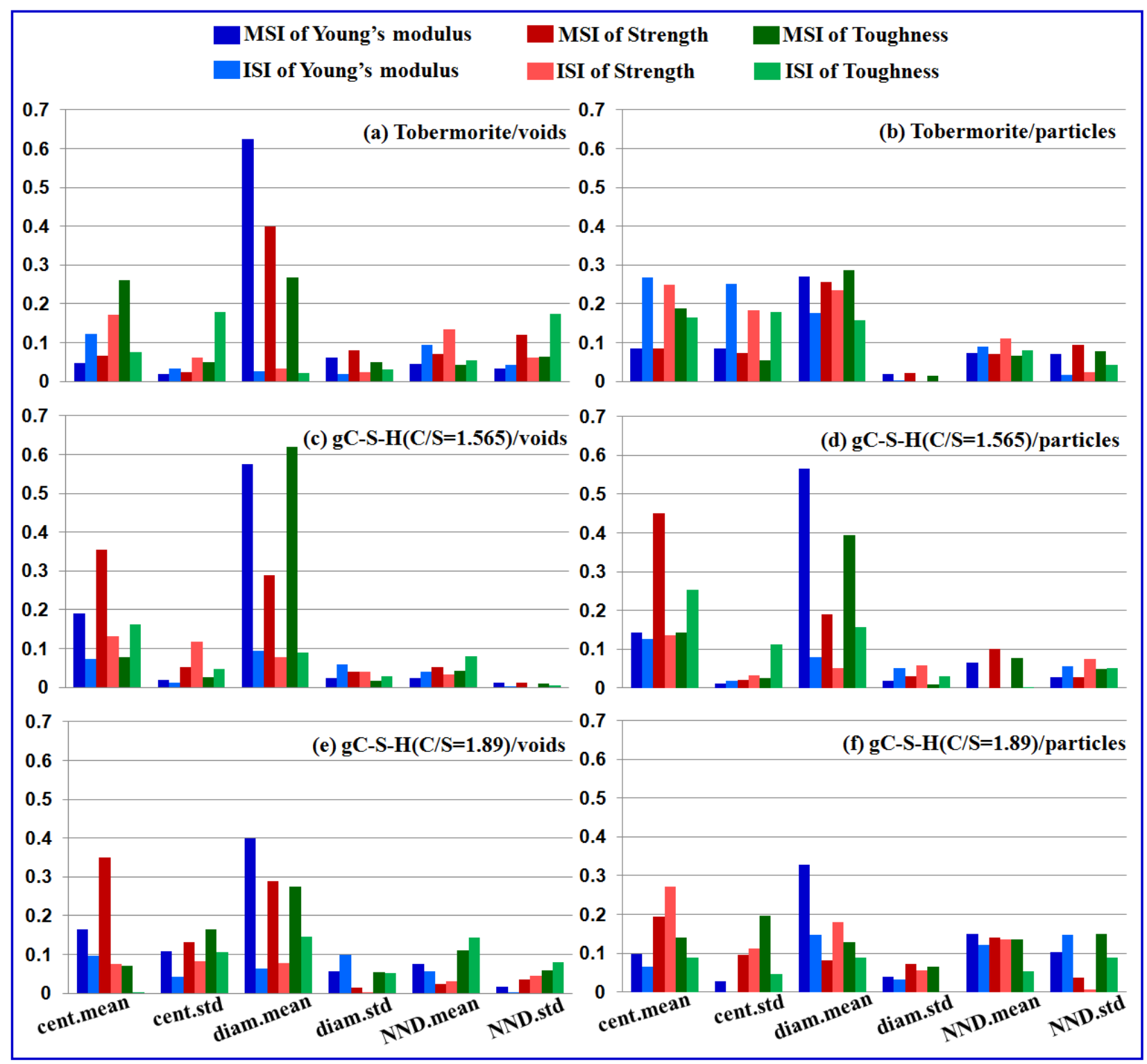

Figure 9. Global sensitivity analysis of main inputs and their two-body cross-interactions with respect to Young's modulus, strength and toughness for interlayer fracture simulations of (a) 
tobermorite, (c) $\mathrm{gC}-\mathrm{S}-\mathrm{H}(\mathrm{C} / \mathrm{S}=1.565)$, (e) $\mathrm{gC}-\mathrm{S}-\mathrm{H}(\mathrm{C} / \mathrm{S}=1.89)$ with random voids; and (b) tobermorite, (d) gC-S-H $(\mathrm{C} / \mathrm{S}=1.565)$, (f) $\mathrm{gC}-\mathrm{S}-\mathrm{H}(\mathrm{C} / \mathrm{S}=1.89)$ with random portlandite particles. The vertical axis indicates the magnitudes of MSI and ISI, which are between 0 and 1.

\subsection{Global sensitivity analysis of tobermorite with random portlandite particles}

For tobermorite with random portlandite particle inclusions, Figure $9 \mathrm{~b}$ shows that the main and interaction effects of diam.mean (V3), as well as the interaction effect of cent.mean (VI), cent.std (V2) and diam.mean are statistically significant input parameters for Young's modulus, strength and toughness. Note that the interaction effects of cent.mean, cent.std and diam.mean make equivalent or even larger contribution to mechanical properties than their main effects. In addition, the main effect of cent.mean (V1 in Table 2) is also statistically important for the toughness. However, compared to tobermorite with random voids, the main effects of the diam.mean to Young's modulus and strength reduce significantly. This suggests the less pronounced role of diam.mean in tobermorite with random portlandite particles. Furthermore, the contribution of NND.std (VO) to toughness is smaller than that of tobermorite with random voids. This might be intuitively expected. NND.std relates to the probability of voids coalescence (a key toughening mechanism here). But unlike voids, portlandite particles do not coalesce during fracture, and thus their NND.std does not matter. Another observation is that the main and interaction effects of diam.std (V4) is almost zero (Figure 9b), which indicates that diam.std has the smallest contribution to mechanical properties. This can be physically understood since portlandite is a solid inclusion in the tobermorite matrix; thus any variation in its diameter would be likely absorbed by the matrix, especially given that both portlandite and tobermorite are rocklike materials with mechanical properties of the same order of magnitude. Overall, although inclusion of solid particles in tobermorite is less sensitive to their variations compared to the 
sensitive role of voids, portlandite particles can still significantly improve the mechanical properties of tobermorite as long as appropriate cent.mean, cent.std and diam.mean are chosen.

\subsection{Global sensitivity analysis of $g C-S-H(C / S=1.565$ and $C / S=1.89)$ with random voids and} portlandite particles

Following the procedure in subsections 4.1 and 4.2 , here we study the global sensitivity analysis of $\mathrm{gC}-\mathrm{S}-\mathrm{H}(\mathrm{C} / \mathrm{S}=1.565$ and 1.89$)$ with random voids and portlandite particles to realize the effect of amorphous character of the matrix. Figure 9c, e show that the main effect of diam.mean (V3 in Table 3, Table 4) is statistically significant for all the studied mechanical properties of gC-S-H with random voids, akin to those of tobermorite. In addition, the main effect of diam.mean of $\mathrm{gC}-\mathrm{S}-\mathrm{H}(\mathrm{C} / \mathrm{S}=1.565$ and 1.89$)$ is the only key parameter to toughness, which is different from the case of tobermorite with random voids. In the latter case the main effects of both cent.mean and diam.mean make significant contributions to toughness, as plotted in Figure 9a. This difference origins from the inherent atomistic structures of tobermorite versus $\mathrm{gC}-\mathrm{S}-\mathrm{H}$. The crystalline structure of tobermorite per se imparts a brittle nature in the material, therefore the main effects of both cent.mean and diam.mean have remarkable effects on the toughness; conceptually the two control the scattering of neighboring voids nearby the central line and thus influence the crack deflection and voids coalescence. On the other hand, the relatively amorphous (glassy) nature of $\mathrm{gC}-\mathrm{S}-\mathrm{H}(\mathrm{C} / \mathrm{S}=1.565$ or 1.89$)$ makes the material inherently disordered and thus already offering multiple paths for crack propagations, diminishing the importance of cent.mean to toughness. In this context, only the main effect of diam.mean stands as the key statistical parameter influencing energy absorption by random voids, and consequently the dominant factor to toughness. Note that in the case of strength, as intuitively expected, the disordered nature of $\mathrm{gC}-\mathrm{S}-\mathrm{H}$ acts in the reverse direction and reduces the yield stress, making $\mathrm{gC}-\mathrm{S}-\mathrm{H}$ vulnerable to 
both cent.mean and diam.mean, hence vital contributions of these two factor to strength. This is in contrast to the behavior of brittle tobermorite where its crystalline nature makes tobermorite's strength insensitive to cent.mean (c.f., cent.mean in Figure 9c, e and Figure 9a). Simply put, the brittle nature of tobermorite (matrix) overshadows the influence of cent.mean of voids to strength.

Similarly, for the gC-S-H with random portlandite particles, it appears that the relative contribution of cent.mean to diam.mean becomes more pronounced in determining the strength (c.f. Figure 9b and Figure 9d, f). Regarding toughness, although diam.mean still plays the dominant role, the interaction effect of cent.mean or the main effect of cent.std becomes important with respect to other nanostructure parameters. This observation implies that the more amorphous feature of gC-S-H make it to go easier through portlandite particles regardless of the restraint of cent.mean or cent.std.

Table 3. Comparison of Main and interaction effects of multiple microstructure parameters on Young's modulus, strength and toughness of $\mathrm{gC}-\mathrm{S}-\mathrm{H}(\mathrm{C} / \mathrm{S}=1.565)$ with random voids and portlandite particles.

\begin{tabular}{|c|c|c|c|c|c|c|c|}
\hline \multirow[t]{2}{*}{ Index } & \multirow[t]{2}{*}{ Input } & \multicolumn{2}{|c|}{$\begin{array}{c}\text { Variance of Young's } \\
\text { modulus (\%) }\end{array}$} & \multicolumn{2}{|c|}{$\begin{array}{l}\text { Variance of strength } \\
\qquad(\%)\end{array}$} & \multicolumn{2}{|c|}{$\begin{array}{c}\text { Variance of toughness } \\
\qquad(\%)\end{array}$} \\
\hline & & Voids & Particles & Voids & Particles & Voids & Particles \\
\hline \multirow{6}{*}{$\begin{array}{l}\text { Main } \\
\text { effect }\end{array}$} & V1 & 12.43 & 8.63 & 18.8 & 20.43 & 5.51 & 2.42 \\
\hline & $\mathrm{V} 2$ & 1.27 & 5.72 & 2.8 & 7.75 & 1.83 & 5.68 \\
\hline & (3) & 38.47 & 20.96 & 15.31 & 16.95 & 43.87) & 9.47 \\
\hline & V4 & 1.48 & 2.99 & 2.09 & 0.82 & 1.21 & 1.82 \\
\hline & V5 & 1.61 & 3.95 & 2.78 & 1.41 & 2.93 & 3.82 \\
\hline & V6 & 0.78 & 0.82 & 0.66 & 2.01 & 0.67 & 2.05 \\
\hline \multirow{4}{*}{$\begin{array}{l}\text { Interaction } \\
\text { effect }\end{array}$} & V1.V2 & 0.22 & 0.74 & 3.45 & 1.65 & 2.02 & 0.02 \\
\hline & V1.V3 & 2.64 & 1.12 & 1.9 & 1.47 & 4.20 & 0.02 \\
\hline & V1.V4 & 1.21 & 2.18 & 0.86 & 0.02 & 1.27 & 0.01 \\
\hline & $\mathrm{V} 1 . \mathrm{V} 5$ & 0.74 & 1.06 & 0.73 & 0.48 & 3.80 & 0.02 \\
\hline
\end{tabular}




\begin{tabular}{l|ll|ll|ll}
\hline V1.V6 & 0.02 & 0.01 & 0.00 & 0.72 & 0.23 & 0.01 \\
V2.V3 & 0.35 & 0.38 & 1.55 & 1.73 & 0.60 & 1.32 \\
V2.V4 & 0.17 & 0.77 & 0.69 & 0.02 & 0.15 & 0.37 \\
V2.V5 & 0.10 & 0.34 & 0.54 & 0.54 & 0.49 & 1.32 \\
V2.V6 & 0.00 & 0.00 & 0.00 & 0.90 & 0.03 & 0.59 \\
V3.V4 & 1.95 & 1.28 & 0.39 & 0.02 & 0.34 & 0.60 \\
V3.V5 & 1.16 & 0.55 & 0.32 & 0.53 & 1.07 & 2.13 \\
V3.V6 & 0.04 & 0.01 & 0.00 & 0.80 & 0.08 & 0.95 \\
V4.V5 & 0.56 & 1.01 & 0.14 & 0.01 & 0.27 & 0.56 \\
V4.V6 & 0.02 & 0.01 & 0.00 & 0.01 & 0.02 & 0.26 \\
V5.V6 & 0.01 & 0.01 & 0.00 & 0.23 & 0.06 & 0.90 \\
\hline
\end{tabular}

Table 4. Comparison of Main and interaction effects of multiple microstructure parameters on Young's modulus, strength and toughness of $\mathrm{gC}-\mathrm{S}-\mathrm{H}(\mathrm{C} / \mathrm{S}=1.89)$ with random voids and portlandite particles.

\begin{tabular}{|c|c|c|c|c|c|c|c|}
\hline \multirow[t]{2}{*}{ Index } & \multirow[t]{2}{*}{ Input } & \multicolumn{2}{|c|}{$\begin{array}{c}\text { Variance of Young's } \\
\text { modulus }(\%)\end{array}$} & \multicolumn{2}{|c|}{$\begin{array}{l}\text { Variance of strength } \\
\qquad(\%)\end{array}$} & \multicolumn{2}{|c|}{$\begin{array}{c}\text { Variance of toughness } \\
\qquad(\%)\end{array}$} \\
\hline & & Voids & Particles & Voids & Particles & Voids & Particles \\
\hline \multirow{6}{*}{$\begin{array}{l}\text { Main } \\
\text { effect }\end{array}$} & V1 & 10.58 & 3.43 & 31.46 & 8.77 & 10.44 & 3.79 \\
\hline & $\mathrm{V} 2$ & 0.86 & 0.96 & 1.38 & 4.27 & 1.91 & 5.30 \\
\hline & & 41.66 & 11.49 & 13.34 & & 29.10 & 3.47 \\
\hline & $\mathrm{V} 4$ & 1.37 & 1.40 & 2.19 & 3.22 & 0.72 & 1.72 \\
\hline & V5 & 4.87 & 5.26 & 7.12 & 6.25 & 5.62 & 3.67 \\
\hline & V6 & 2.02 & 3.59 & 2.00 & 1.66 & 3.54 & 4.01 \\
\hline \multirow{10}{*}{$\begin{array}{l}\text { Interaction } \\
\text { effect }\end{array}$} & V1.V2 & 0.71 & 0.00 & 1.34 & 2.63 & 5.71 & 0.35 \\
\hline & V1.V3 & 3.77 & 0.79 & 2.09 & 4.85 & 8.69 & 0.81 \\
\hline & V1.V4 & 2.25 & 0.12 & 2.60 & 1.22 & 1.57 & 0.00 \\
\hline & V1.V5 & 0.00 & 0.59 & 0.00 & 3.40 & 0.05 & 0.42 \\
\hline & V1.V6 & 2.58 & 0.79 & 3.49 & 0.11 & 2.56 & 0.81 \\
\hline & $\mathrm{V} 2 . \mathrm{V} 3$ & 0.30 & 0.00 & 0.27 & 1.16 & 1.79 & 0.35 \\
\hline & $\mathrm{V} 2 . \mathrm{V} 4$ & 0.15 & 0.00 & 0.33 & 0.36 & 0.24 & 0.00 \\
\hline & V2.V5 & 0.00 & 0.00 & 0.00 & 0.81 & 0.01 & 0.18 \\
\hline & V2.V6 & 0.16 & 0.00 & 0.39 & 0.03 & 0.53 & 0.35 \\
\hline & $\mathrm{V} 3 . \mathrm{V} 4$ & 0.86 & 0.36 & 0.48 & 0.56 & 0.35 & 0.00 \\
\hline
\end{tabular}




\begin{tabular}{l|ll|ll|ll}
\hline V3.V5 & 0.00 & 1.71 & 0.00 & 1.46 & 0.01 & 0.42 \\
V3.V6 & 0.90 & 2.33 & 0.69 & 0.05 & 0.64 & 0.82 \\
V4.V5 & 0.00 & 0.27 & 0.00 & 0.39 & 0.00 & 0.00 \\
V4.V6 & 0.50 & 0.36 & 0.70 & 0.01 & 0.12 & 0.00 \\
V5.V6 & 0.00 & 1.69 & 0.00 & 0.03 & 0.00 & 0.42 \\
\hline
\end{tabular}

Comparing gC-S-H with $\mathrm{C} / \mathrm{S}=1.56$ and $\mathrm{C} / \mathrm{S}=1.89$ with void or partlandite particles (Figure $9 \mathrm{c}-\mathrm{f}$ ), the effect of both NND.mean and NND.std become more pronounced for the $\mathrm{gC}-\mathrm{S}-\mathrm{H}$ with $\mathrm{C} / \mathrm{S}=1.89$. This can be conceptually understood since the structure with $\mathrm{C} / \mathrm{S}=1.89$ is inherently more defective than the one with $\mathrm{C} / \mathrm{S}=1.56$. This inherent randomness of the matrix facilitates the coalescence of the neighboring voids/particles inclusions. Another interesting observation between the two gC-S-H structures is that the diam.mean has a more dominant role in the case of $\mathrm{C} / \mathrm{S}=1.56$ versus $\mathrm{C} / \mathrm{S}=1.89$ (Figure 9c-f). Although both structures have an amorphous-like structure, the one with less defects $(\mathrm{C} / \mathrm{S}=1.56)$ is more vulnerable to the average size of void/particle inclusions. This in part could be also due to the higher density of C-S-H with $\mathrm{C} / \mathrm{S}=1.56$, which is higher than both tobermorite and C-S-H with $\mathrm{C} / \mathrm{S}=1.89$. In brief, given that the real hydrated Portland cement has a more frequency of C-S-H phases with $\mathrm{C} / \mathrm{S}$ around 1.61.7 (Pellenq, Kushima et al. 2009), from practical standpoints our study suggests that it is preferred to control the diameter of void/particle inclusions within a desired mean value, rather than their standard deviations or their locations (NND). Further detailed analyses on this and possible correlations to the medium range defect attribute of combinatorial C-S-H with various C/S ratios (Shahsavari, Qomi, Krakowiak et al. 2014) and potential defects (Shahsavari and Chen 2015, Shahsavari, Tao et al. 2016) will be subjects of future studies. 


\section{Conclusion}

We studied the effect of random nanovoids and particle inclusions on balancing mechanical properties (strength, stiffness and toughness) of a complex cementitious phase, calcium-silicatehydrate $(\mathrm{C}-\mathrm{S}-\mathrm{H})$, with varying stoichiometry and molecular morphology. By performing over $600 \mathrm{MD}$ simulations coupled with statistical analysis tools, our results provide new fundamental understanding on fracture mechanisms associated with $\mathrm{C}-\mathrm{S}-\mathrm{H}$ at the molecular scale, particularly the interplay between the crack tip, nanovoid/particle inclusions, and the extent of crystallinity of the matrix, which relates to its stoichiometry. In the first part of this work, our key finding elucidate ductile fracture mechanisms associated with C-S-H. In particular,

1. In all $\mathrm{C}-\mathrm{S}-\mathrm{H}$ phases including crystalline tobermorite and amorphous $\mathrm{gC}-\mathrm{S}-\mathrm{H}(\mathrm{C} / \mathrm{S}=1.565$ and 1.89), toughness increases with introduction of voids and portlandite particles, owing to competing mechanisms such as crack deflection, voids coalescence, internal necking, accommodation, and geometry alteration of individual voids/particles. These phenomena are reminiscent of ductile fracture mechanisms of crystalline alloys and ductile metals (Scheyvaerts, Pardoen et al. 2009, Benzerga and Leblond 2010, Glezer, Shurygina et al. 2013), providing evidence that complex macroscale brittle materials such as C-S-H may exhibit ductile fracture mechanisms at the nanoscale.

2. Considering the inter- or intra-layer fracture of crystalline tobermorite, addition of portlandite particle inclusions causes a brittle-to-ductile transition, leading to significantly improved fracture strain and toughness while exhibiting a modest sacrifice in strength and Young's modulus. This is because crack deflection and geometry alteration of particles absorb more energy during crack propagation, contributing to toughness. 
3. Due to the distortion of silica chains and amorphization of the overall structure, inclusion of nanovoids and portlandite particles in $\mathrm{gC}-\mathrm{S}-\mathrm{H}$ with $\mathrm{C} / \mathrm{S}=1.565$ and 1.89 shows more ductile behavior compared to crystalline tobermorite with $\mathrm{C} / \mathrm{S}=1$. New crack nucleation from the edges of voids and particles, along with the subsequent deflection of propagation path are responsible for this behavior.

4. In the case of $\mathrm{gC}-\mathrm{S}-\mathrm{H}$ with $\mathrm{C} / \mathrm{S}=1.89$, addition of portlandite particles will not only increase the toughness, but also the strength of composite because the amorphous structure induces strong chemical interactions such as H-bonds between C-S-H and portlandite molecules.

5. Under a given loading condition, crack propagation path depends essentially on the microstructural design. Considering the complex amorphous characteristic of C-S-H, controlling the crack-growth mechanisms associated with deflection, bypassing, and cracktip blunting may enable reliable guidelines in controlling the crack growth behavior, leading to optimum mechanical properties $\mathrm{C}-\mathrm{S}-\mathrm{H}$ and thus concrete.

In the second part, we performed a comprehensive global sensitivity analysis up to the second order to unravel the importance of random nanostructural features on mechanical properties. In particular, due to the stochastic nature of crack growth, combined with the random location and size of voids and particle inclusions, and the complex chemistry of the underlying matrix (which dictates the extent of amorphous vs. crystalline features of C-S-H), we considered six key input random variables (Table 1), leading to the following conclusions:

6. The mean diameter (diam.mean) of voids/particles is a critical parameter determining the mechanical properties of $\mathrm{C}-\mathrm{S}-\mathrm{H}$, irrespective of $\mathrm{C} / \mathrm{S}$ ratios or crystalline versus amorphous features of the substrates. The larger the diam.mean, the lower the strength and Young's 
modulus. Toughness behavior is more complicated and can be influenced by other random parameters as well.

7. While the contribution of the main effects of individual input parameters are generally larger than their cross-interactions effects, there are situations where the secondary crossinteractions effects are more important that the main effects. For instance, the interaction effect of standard deviation of central distance (cent.std) and standard deviation of nearest neighbor distance (NND.std) in toughness of crystalline tobermorite with random voids is more important than the individual effects of cent.std or NND.std. Thus, modulating these two parameters, which together control the scattering of neighboring voids nearby the central crack line, will significantly impact the crack propagation path and thus toughness.

8. Both the main and interaction effects of standard deviation of diameter (diam.std) have negligible impact on mechanic of crystalline tobermorite with portlandite particle inclusions. This is because portlandite is a solid inclusion in a solid tobermorite matrix; any variation in its diameter would be absorbed/filled by the matrix. However, in the case of amorphous gC$\mathrm{S}-\mathrm{H}$ matrix $(\mathrm{C} / \mathrm{S}=1.56$ or 1.89$)$, the effect of diam.std becomes more pronounced.

9. While both mean of central distance (cent.mean) and mean of diameter (diam.mean) control the toughness of crystalline tobermorite with voids/particles, in the case of amorphous gC-S$\mathrm{H}$ (either $\mathrm{C} / \mathrm{S}=1.56$ or 1.89 ), toughness is mainly influenced by diam.mean. In brittle tobermorite, cent.mean is a key to crack deflection and void coalescence whereas the relatively amorphous (glassy) nature of $\mathrm{gC}-\mathrm{S}-\mathrm{H}$ matrix $(\mathrm{C} / \mathrm{S}=1.565$ or 1.89$)$ offers multiple paths for crack propagations, diminishing the importance of cent.mean to toughness.

10. In contrast to toughness, strength has a reverse dependence on random parameters of nanovoids/particles in crystalline tobermorite versus amorphous $\mathrm{gC}-\mathrm{S}-\mathrm{H}$, i.e. both cent.mean 
and diam.mean contribute to the strength of $\mathrm{gC}-\mathrm{S}-\mathrm{H}(\mathrm{C} / \mathrm{S}=1.56$ or 1.89$)$ whereas brittle nature of tobermorite overshadows the impact of cent.mean.

11. Comparing the two $\mathrm{gC}-\mathrm{S}-\mathrm{H}(\mathrm{C} / \mathrm{S}=1.56$ vs. 1.89$)$ with voids/particles, the effects of both mean of nearest neighbor distance (NND.mean) and standard deviation of nearest neighbor distance (NND.std) become more pronounced for $\mathrm{gC}-\mathrm{S}-\mathrm{H}$ with $\mathrm{C} / \mathrm{S}=1.89$, since its higher defective sites facilitate coalescence of neighboring voids/particles. Furthermore, gC-S-H with $\mathrm{C} / \mathrm{S}=1.56$ is more vulnerable to mean of diameter (diam.mean), in part due to its less defective sites and in part due to more density of $\mathrm{C} / \mathrm{S}=1.56$ among all $\mathrm{C}-\mathrm{S}-\mathrm{H}$ polymorphs (Qomi, Krakowiak et al. 2014). Given that the $\mathrm{C} / \mathrm{S}=1.56$ is closer to the average stoichiometry of typical Portland cement, our study suggests that controlling average diameter of porlandite particles in cement paste is more important than their standard deviations or their spatial locations (nearest neighbor distance/NND).

Above conclusions have limitations too. First, we considered a 2D model with parallel and cylindrical voids or portlandite inclusions as a first approximation to a real 3D system. Although such as $2 \mathrm{D}$ model is reasonable for a platelet-like C-S-H structure, a full 3D simulations must be carried out in the future. Second, the focus of this work was on voids and portlandite particles within an "individual C-S-H grain". At such a nanometer scale, the C-S-H with voids and particles still maintain its crystalline structure, however, in reality (larger scales) there might be alterations to the crystallinity of the matrix. Finally, our simulation results are obtained based on single crystalline $\mathrm{C}-\mathrm{S}-\mathrm{H}$ at the order of a few hundred nanometers while real cementitious materials have a hierarchical structure (Shahsavari 2011), spanning from subnano to micro or macrometer length scales. This multi-scale characteristic calls for an in-depth study on deformation mechanisms at different scales, which must be thoroughly conducted in the future. 
In this context, our nanoscale data will provide the key necessary information at the smallest scales, which affect mechanics at larger scales. We recall that crack growth and strength is an inherent property, which is controlled by nanoscale deformation mechanisms. This original study at the nanometer length scale is the first report on the fundamental molecular-level properties, which impact the mechanical properties at larger scales. Our data will provide new insights, design guidelines, and strategies to turn the brittle cement paste (C-S-H) into a ductile material, impacting the manufacturing of concrete infrastructures and potentially other complex brittle materials and composites.

\section{Acknowledgements}

We acknowledge support from National Science Foundation (NSF) Grant Nos. CMMI-1235522. The use of supercomputer machines for this work was supported in part by National Insitutes of Health Award No. NCRR S10RR02950 and an IBM Shared University Research (SUR) Award in partnership with CISCO, Qlogic, and Adaptive Computing, and in part by the Data Analysis and Visualization Cyber infrastructure funded by NSF under Grant No. OCI-0959097.

\section{References}

Alarcon-Ruiz, L., G. Platret, E. Massieu and A. Ehrlacher (2005). "The use of thermal analysis in assessing the effect of temperature on a cement paste." Cement and Concrete Research 35(3): 609-613.

Allen, A. J., J. J. Thomas and H. M. Jennings (2007). "Composition and density of nanoscale calcium-silicate-hydrate in cement." Nature Materials 6(4): 311-316. 
Bauchy, M., H. Laubie, M. A. Qomi, C. Hoover, F.-J. Ulm and R.-M. Pellenq (2015). "Fracture toughness of calcium-silicate-hydrate from molecular dynamics simulations." Journal of NonCrystalline Solids 419: 58-64.

Bauchy, M., M. J. A. Qomi, C. Bichara, F. J. Ulm and R. J. M. Pellenq (2014). "Nanoscale Structure of Cement: Viewpoint of Rigidity Theory." Journal of Physical Chemistry C 118(23): 12485-12493.

Benzerga, A. and J.-B. Leblond (2010). "Ductile fracture by void growth to coalescence." Advances in Applied Mechanics 44: 169-305.

Benzerga, A. A. and J. B. Leblond (2010). Ductile Fracture by Void Growth to Coalescence. Advances in Applied Mechanics, Vol 44. H. Aref and E. VanDerGiessen. San Diego, Elsevier Academic Press Inc. 44: 169-305.

Birchall, J., A. Howard and K. Kendall (1981). "Flexural strength and porosity of cements."

Bordallo, H. N., L. P. Aldridge and A. Desmedt (2006). "Water dynamics in hardened ordinary Portland cement paste or concrete: From quasielastic neutron scattering." Journal of Physical Chemistry B 110(36): 17966-17976.

Chamrova, R. (2010). "Modelling and measurement of elastic properties of hydrating cement paste."

Chen, J. J., L. Sorelli, M. Vandamme, F. J. Ulm and G. Chanvillard (2010). "A Coupled Nanoindentation/SEM- EDS Study on Low Water/Cement Ratio Portland Cement Paste: Evidence for $\mathrm{C}-\mathrm{S}-\mathrm{H} / \mathrm{Ca}(\mathrm{OH}) 2$ Nanocomposites." Journal of the American Ceramic Society 93(5): 1484-1493. 
Chindaprasirt, P., C. Jaturapitakkul and T. Sinsiri (2005). "Effect of fly ash fineness on compressive strength and pore size of blended cement paste." Cement and Concrete Composites 27(4): 425-428.

Cong, X. and R. J. Kirkpatrick (1996). "29 Si MAS NMR study of the structure of calcium silicate hydrate." Advanced Cement Based Materials 3(3): 144-156.

Dai, W., Z.-H. Shui and K. Li (2012). "Molecular Dynamics Simulation on Amorphous Calcium Silicate Hydrate System." Advanced Science Letters 5(2): 710-713.

Dolado, J. S., M. Griebel, J. Hamaekers and F. Heber (2011). "The nano-branched structure of cementitious calcium-silicate-hydrate gel." Journal of Materials Chemistry 21(12): 4445-4449. Feldman, R. F. and P. J. Sereda (1968). "A model for hydrated Portland cement paste as deduced from sorption-length change and mechanical properties." Materiaux et construction 1(6): 509520.

Galmarini, S., A. Aimable, N. Ruffray and P. Bowen (2011). "Changes in portlandite morphology with solvent composition: Atomistic simulations and experiment." Cement and Concrete Research 41(12): 1330-1338.

Glezer, A., N. Shurygina, S. Zaichenko and I. Permyakova (2013). "Interaction of deformation shear bands with nanoparticles in amorphous-nanocrystalline alloys." Russian Metallurgy (Metally) 2013(4): 235-244.

Gonzalez-Teresa, R., J. S. Dolado, A. Ayuela and J. C. Gimel (2013). "Nanoscale texture development of C-S-H gel: A computational model for nucleation and growth." Applied Physics Letters 103(23). 
Greener, J., H. Peemoeller, C. H. Choi, R. Holly, E. J. Reardon, C. M. Hansson and M. M. Pintar (2000). "Monitoring of hydration of white cement paste with proton NMR spin-spin relaxation." Journal of the American Ceramic Society 83(3): 623-627.

Hufnagel, T., C. Fan, R. Ott, J. Li and S. Brennan (2002). "Controlling shear band behavior in metallic glasses through microstructural design." Intermetallics 10(11): 1163-1166.

Ioannidou, K., K. J. Krakowiak, M. Bauchy, C. G. Hoover, E. Masoero, S. Yip, F.-J. Ulm, P. Levitz, R. J.-M. Pellenq and E. Del Gado (2016). "Mesoscale texture of cement hydrates." Proceedings of the National Academy of Sciences 113(8): 2029-2034.

Jalilvand, S. and R. Shahsavari (2015). "Molecular Mechanistic Origin of Nanoscale Contact, Friction, and Scratch in Complex Particulate Systems." Acs Applied Materials \& Interfaces 7(5): 3362-3372.

Janik, J. A., W. Kurdowski, R. Podsiadly and J. Samseth (2001). "Fractal structure of C-S-H and tobermorite phases." Acta Physica Polonica A 100(4): 529-537.

Jennings, H. M. (2008). "Refinements to colloid model of C-S-H in cement: CM-II." Cement and Concrete Research 38(3): 275-289.

Kim, J.-J., Y. Choi, S. Suresh and A. Argon (2002). "Nanocrystallization during nanoindentation of a bulk amorphous metal alloy at room temperature." Science 295(5555): 654-657.

Lewandowski, J. J. and A. L. Greer (2006). "Temperature rise at shear bands in metallic glasses." Nature Materials 5(1): 15-18.

Maekawa, K., Tetsuya Ishida, and Toshiharu Kishi. (2003). "Multi-scale modeling of concrete performance." Journal of Advanced Concrete Technology 1(2): 91-126.

Manzano, H., E. Masoero, I. Lopez-Arbeloa and H. M. Jennings (2013). "Shear deformations in calcium silicate hydrates." Soft Matter 9(30): 7333-7341. 
Manzano, H., S. Moeini, F. Marinelli, A. C. T. van Duin, F. J. Ulm and R. J. M. Pellenq (2012).

"Confined Water Dissociation in Microporous Defective Silicates: Mechanism, Dipole Distribution, and Impact on Substrate Properties." Journal of the American Chemical Society 134(4): 2208-2215.

Mehta, P. K. (2001). "Reducing the environmental impact of concrete." Concrete international 23(10): 61-66.

Meral, C., C. Benmore and P. J. Monteiro (2011). "The study of disorder and nanocrystallinity in $\mathrm{C}-\mathrm{S}-\mathrm{H}$, supplementary cementitious materials and geopolymers using pair distribution function analysis." Cement and Concrete Research 41(7): 696-710.

Nose, S. (1984). "A UNIFIED FORMULATION OF THE CONSTANT TEMPERATURE MOLECULAR-DYNAMICS METHODS." Journal of Chemical Physics 81(1): 511-519.

Pellenq, R. J. M., A. Kushima, R. Shahsavari, K. J. Van Vliet, M. J. Buehler, S. Yip and F. J. Ulm (2009). "A realistic molecular model of cement hydrates." Proceedings of the National Academy of Sciences of the United States of America 106(38): 16102-16107.

Plimpton, S. (1995). "FAST PARALLEL ALGORITHMS FOR SHORT-RANGE MOLECULAR-DYNAMICS." Journal of Computational Physics 117(1): 1-19.

Qomi, M. J. A., K. J. Krakowiak, M. Bauchy, K. L. Stewart, R. Shahsavari, D. Jagannathan, D. B. Brommer, A. Baronnet, M. J. Buehler, S. Yip, F. J. Ulm, K. J. Van Vliet and R. J. M. Pellenq (2014). "Combinatorial molecular optimization of cement hydrates." Nature Communications 5.

Rafiee, M. A., T. N. Narayanan, D. P. Hashim, N. Sakhavand, R. Shahsavari, R. Vajtai and P. M. Ajayan (2013). "Hexagonal Boron Nitride and Graphite Oxide Reinforced Multifunctional Porous Cement Composites." Advanced Functional Materials: 5624-5630. 
Ritchie, R. O. (2011). "The conflicts between strength and toughness." Nature Materials 10(11): 817-822.

Sakhavand, N., P. Muthuramalingam and R. Shahsavari (2013). "Toughness Governs the Rupture of the Interfacial H-Bond Assemblies at a Critical Length Scale in Hybrid Materials." Langmuir 29(25): 8154-8163.

Sakhavand, N. and R. Shahsavari (2014). "Synergistic Behavior of Tubes, Junctions and Sheets Imparts Mechano-Mutable Functionality in 3D Porous Multifunctional Boron Nitride Nanostructures." Journal of Physical Chemistry C 118(39): 22730-22738.

Sakhavand, N. and R. Shahsavari (2015). "Universal composition-structure-property maps for natural and biomimetic platelet-matrix composites and stacked heterostructures." Nature Communications 6.

Scheyvaerts, F., T. Pardoen and P. Onck (2009). "A new model for void coalescence by internal necking." International Journal of Damage Mechanics.

Seppala, E. T., J. Belak and R. E. Rudd (2004). "Onset of void coalescence during dynamic fracture of ductile metals." Physical Review Letters 93(24).

Shahsavari, R. "Hierarchical Modeling of Structure and Mechanics of Cement Hydrate, Ph.D Thesis, Cambridge, MIT (2010)."

Shahsavari, R. (2011). Hierarchical modeling of structure and mechanics of cement hydrate, Massachusetts Institute of Technology.

Shahsavari, R., M. J. Buehler, R. J. M. Pellenq and F. J. Ulm (2009). "First-Principles Study of Elastic Constants and Interlayer Interactions of Complex Hydrated Oxides: Case Study of Tobermorite and Jennite." Journal of the American Ceramic Society 92(10): 2323-2330. 
Shahsavari, R. and L. Chen (2015). "Screw Dislocations in Complex, Low Symmetry Oxides: Core Structures, Energetics and Impact on Crystal Growth." ACS Applied Materials and Interfaces 7(4): 2223-2234.

Shahsavari, R., R. J. M. Pellenq and F. J. Ulm (2011). "Empirical force fields for complex hydrated calcio-silicate layered materials." Physical Chemistry Chemical Physics 13(3): 10021011.

Shahsavari, R., L. Tao and L. Chen (2016). "Structure, Energetics, and Impact of Screw Dislocations in Tricalcium Silicates." Journal of the American Ceramic Society: n/a-n/a.

Skinner, L. B., S. R. Chae, C. J. Benmore, H. R. Wenk and P. J. M. Monteiro (2010). "Nanostructure of Calcium Silicate Hydrates in Cements." Physical Review Letters 104(19).

Ulm, F., Z. Bazant and F. Wittman (2001). Creep, Shrinkage, and Durability Mechanics of Concrete and Other Quasi-Brittle Materials, Elsevier.

Vangunsteren, W. F. and H. J. C. Berendsen (1990). "COMPUTER-SIMULATION OF MOLECULAR-DYNAMICS - METHODOLOGY, APPLICATIONS, AND PERSPECTIVES IN CHEMISTRY." Angewandte Chemie-International Edition in English 29(9): 992-1023.

Verlet, L. (1967). "COMPUTER EXPERIMENTS ON CLASSICAL FLUIDS .I. THERMODYNAMICAL PROPERTIES OF LENNARD-JONES MOLECULES." Physical Review 159(1): 98-\&.

Weck, A., D. S. Wilkinson, E. Maire and H. Toda (2008). "Visualization by X-ray tomography of void growth and coalescence leading to fracture in model materials." Acta Materialia 56(12): 2919-2928.

Woodcock, L. V. (1971). "ISOTHERMAL MOLECULAR DYNAMICS CALCULATIONS FOR LIQUID SALTS." Chemical Physics Letters 10(3): 257-\&. 
Worrell, E., L. Price, N. Martin, C. Hendriks and L. O. Meida (2001). "Carbon dioxide emissions from the global cement industry 1." Annual review of energy and the environment 26(1): 303329.

Yin, X., W. Chen, A. To, C. McVeigh and W. K. Liu (2008). "Statistical volume element method for predicting microstructure-constitutive property relations." Computer methods in applied mechanics and engineering 197(43): 3516-3529.

Zhang, N. and Y. Chen (2012). "Molecular origin of the sawtooth behavior and the toughness of nacre." Materials Science and Engineering: C 32(6): 1542-1547.

Zhang, N. and Y. P. Chen (2013). "Nanoscale plastic deformation mechanism in single crystal aragonite." Journal of Materials Science 48(2): 785-796.

Zhang, N., Q. A. Deng, Y. Hong, L. M. Xiong, S. Li, M. Strasberg, W. Q. Yin, Y. J. Zou, C. R. Taylor, G. Sawyer and Y. P. Chen (2011). "Deformation mechanisms in silicon nanoparticles." Journal of Applied Physics 109(6). 